\title{
Identities on Hyperbolic Manifolds
}

\author{
Martin Bridgeman* and Ser Peow Tan** \\ Boston College \\ Chestnut Hill, Ma 02116, USA \\ email: bridgem@bc.edu \\ National University of Singapore \\ 10 Lower Kent Ridge Road, S(119076), Singapore \\ email:mattansp@nus.edu.sg
}

\begin{abstract}
In this survey, we discuss four classes of identities due principally to Basmajian, McShane, Bridgeman-Kahn and Luo-Tan on hyperbolic manifolds and provide a unified approach for proving them. We also elucidate on the connections between the various identities.
\end{abstract}

2000 Mathematics Subject Classification:

Keywords: Hyperbolic manifolds, identities, orthogeodesic, ortholength, orthospectrum, simple geodesics, geodesic flow.

\section{Introduction}

In the last couple of decades, several authors have discovered various remarkable and elegant identities on hyperbolic manifolds, including Basmajian [3, McShane [32, 33, Bridgeman-Kahn [11, 13] and Luo-Tan [28]. Some of these identities have been generalized and extended, with different and independent proofs given in some cases. In addition to their intrinsic beauty and curiosity value, some of the identities have found important applications, in particular, the McShane identity as generalized by Mirzakhani to surfaces with boundary played a crucial role in Mirzakhani's computation of the Weil-Petersson volumes of the moduli spaces of bordered Riemann surfaces, as well as some subsequent applications; the Bridgeman-Kahn identity gave lower bounds for

\footnotetext{
* This work was partially supported by a grant from the Simons Foundation (\#266344 to Martin Bridgeman)

**Tan was partially supported by the National University of Singapore academic research grant R-146-000-156-112.
} 
the volumes of hyperbolic manifolds with totally geodesic boundary. Many of these identities were proven independently of each other, for example, although the Basmajian and McShane identities appeared at about the same time, the authors seemed unaware of each other's work at that point; the Bridgeman-Kahn identity, although it appeared later was also proven somewhat independently of both of these works. The exception is the Luo-Tan identity which borrowed inspiration from the previous works.

The aim of this article is to explore these identities, to analyse the various ingredients which make them work, and to provide a common framework for which to understand them. We hope that this will not only put the identities in a more natural setting and make them easier to understand, but also point the way towards a more unified theory with which to view these beautiful identities, and also point the direction towards possible applications for these identities.

The unifying idea behind all of these identities is fairly simple. One considers a set $X$ with finite measure $\mu(X)$ associated with the hyperbolic manifold $M$, (for example $X=\partial M$, the boundary of $M$, or $X=T_{1}(M)$, the unit tangent bundle of $M$ ) and look for interesting geometric/dynamical/measure theoretic decompositions of $X$. Typically, by exploring some geometric or dynamical aspect of $M$, one can show that the set $X$ decomposes into a countable union of disjoint subsets $X_{i}$ of finite non-zero measure, and a set $Z$ which is geometrically and dynamically complicated and interesting, but which has measure zero (this is what we mean by a measure theoretic decomposition of $X)$. One deduces the fact that $Z$ has measure zero from a deep but well-known result from hyperbolic geometry or dynamical systems. The identity is then just the tautological equation

$$
\mu(X)=\sum_{i} \mu\left(X_{i}\right) .
$$

The second part of the problem consists of analysing the sets $X_{i}$, in particular, to compute their measures in terms of various geometric quantities like spectral data. This can be relatively simple, for example in the case of the Basmajian identity, somewhat more complicated, like the McShane and Bridgeman-Kahn identities or considerably more involved, as in the Luo-Tan identities. A typical feature is that the sets $X_{i}$ are indexed by either simple geometric objects on $M$ like orthogeodesics, or simple subsurfaces of $M$, like embedded one-holed tori or thrice-punctured spheres. In particular, their measures depend only on the local geometry and data, and not on the global geometry of $M$.

One can develop this viewpoint further, for example letting $X$ be the set of geodesics on $M$ with the Liouville measure and associating to $X$ the length of the geodesic as a random variable and computing the moment generating function of this. In this way, for example, the Basmajian and the Bridgeman- 
Kahn identities can be viewed as different moments of the same generating function, see [14.

Alternatively, as in the case of Bowditch's proof 8 of McShane's original identity, one can adopt a different viewpoint, and prove it using a combination of algebraic and combinatorial techniques. This has been developed further in [9, 10, 46, 47, 49] etc, and provides an interesting direction for further exploration.

Our main aim in this survey is to demystify these identities and to show that the basic ideas involved in deriving them are very simple. As such, the exposition will be somewhat leisurely, and where necessary, we will present slightly different proofs and perspectives than the original papers. We will refer the reader to the original papers for the more technical details of computing the measures $\mu\left(X_{i}\right)$.

The rest of the paper is organized as follows. In the next couple of sections we first state the four sets of identities, and then sketch the proofs for these identities from our perspective. Subsequently, we give a short discussion of the moment point of view adopted in 14] which allows one to view the Basmajian and Bridgeman identities as different moments of the same variable and follow this with a short discussion of the Bowditch proof of the McShane identity and subsequent developments. We conclude the survey with some open questions and directions for further investigations.

\subsection{Literature}

The literature on the subject is fairly large and growing. To aid the reader we will now give a brief synopsis by identity.

McShane Identity: The McShane identity first appeared in McShane's 1991 thesis [32, "A remarkable identity for lengths of curves". This was subsequently generalized (to higher genus surfaces) and published in [33. In the papers [8, 9, 10], Bowditch gives a proof of the McShane identity using Markov triples, with extensions to punctured torus bundles and type-preserving quasifuchsian representations, see also 1, 2] by Akiyoshi, Miyachi and Sakuma for variations. The identity was extended to surfaces with cone singularities in Zhang's 2004 thesis [51, see also [45. A Weierstrass points version of the identity was derived by McShane in [34, and the identity was also generalized to closed surfaces of genus two in [35], using similar techniques. Both of these can also be derived using the hyperelliptic involution on the punctured torus and on a genus two surface, and using the identity on the resulting cone surfaces, as explained by Tan, Wong and Zhang in [45. Mirzakhani gave a proof of the general McShane identity for bordered surface in her 2005 thesis [36] which was subsequently published in [37. In [46, the identity was generalized to the $\operatorname{SL}(2, \mathbb{C})$ case by Tan, Wong and Zhang and for non-orientable surfaces 
by Norbury in $[39$. The identity was generalized to $\operatorname{PSL}(n, \mathbb{R})$ for Hitchin representations by Labourie and McShane in 24. A version for two-bridge links was given by Lee and Sakuma in 26. Recent work of $\mathrm{Hu}$, Tan and Zhang in 21, 22] have also given new variations and extensions of the identity to the context of Coxeter group actions on $\mathbb{C}^{n}$.

Basmajian Identity: The Basmajian identity appears in the 1993 paper [3], "The orthogonal spectrum of a hyperbolic manifold". A recent paper of Vlamis [50] analyses the statistics of the Basmajian identity and derives a formula for the moments of its associated hitting random variable. In the recent paper [41, Paulin and Parkkonen derive formulae for the asymptotic distribution of orthogonal spectrum in a general negatively curved space.

Bridgeman-Kahn Identity: The Bridgeman-Kahn identity was first proven in the surface case by the first author in the 2011 paper [11] "Orthospectra and Dilogarithm Identities on Moduli Space". An alternate proof was given by Calegari in [15]. The general case was proven by Bridgeman-Kahn in the paper [13]. The paper [16] of Calegari analyses the connections between the Bridgeman-Kahn identity and Basmajian identity and gives an orthospectrum identity that has the same form as the Bridgeman-Kahn identity but arises out of a different decomposition. A recent paper of Masai and McShane [30] has shown that the identity obtained by Calegari is in fact the original Bridgeman-Kahn identity. In the paper [14] the authors consider the statistics of the Bridgeman-Kahn identity and derive a formula for the moments of its associated hitting random variable. We show that the Basmajian and Bridgeman-Kahn identities arise as the first two moments of this random variable.

Luo-Tan identity: The Luo-Tan identity appears in the 2011 preprint 28] "A dilogarithm identity on Moduli spaces of curves". A version of the identity for small hyperbolic surfaces can be found in [20] and for surfaces with boundary and non-orientable surfaces in 29 .

Acknowledgements. We are grateful to Dick Canary, Francois Labourie, Feng Luo, Greg McShane, Hugo Parlier, Caroline Series, and Ying Zhang for helpful discussions on this material.

\section{Two orthospectra identities}

We let $M$ be a finite volume hyperbolic manifold with non-empty totally geodesic boundary. In [3], Basmajian introduced the notion of orthogeodesics for hyperbolic manifolds. An orthogeodesic $\alpha$ for $M$ is an oriented proper geodesic arc in $M$ which is perpendicular to $\partial M$ at its endpoints (see figure 
1). Let $O_{M}$ be the collection of orthogeodesics for $M$ and $L_{M}$ the set of lengths of orthogeodesics (with multiplicity). The set $L_{M}$ is the ortholength spectrum, note that all multiplicities are even since we consider oriented orthogeodesics.

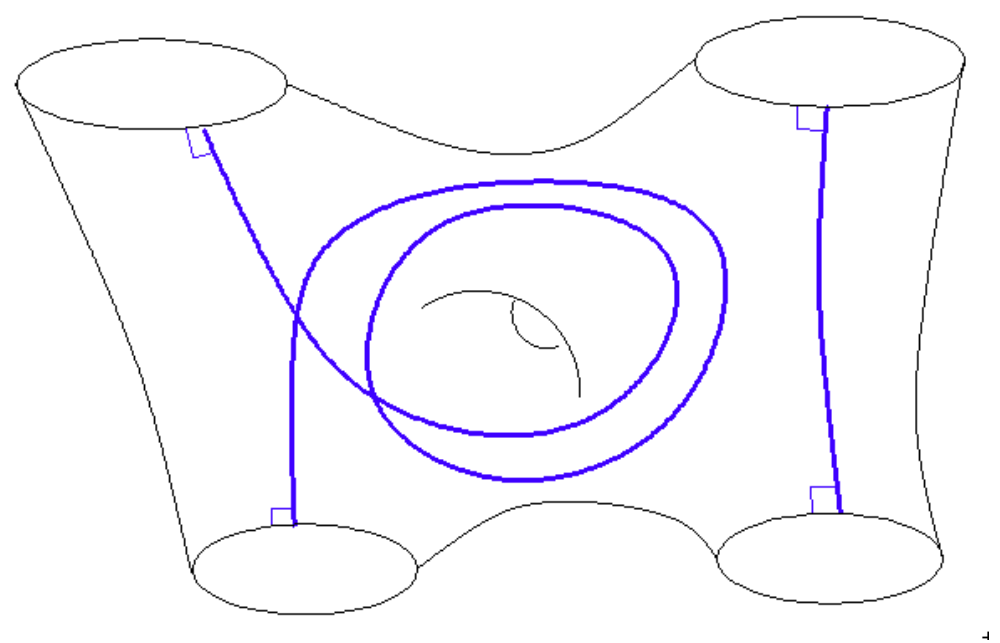

Figure 1. Two orthogeodesics, one simple, one non-simple

\subsection{Basmajian Identity}

In the 1993 paper [3], The orthogonal spectrum of a hyperbolic manifold, Basmajian derived the following orthospectrum identity:

Theorem 2.1. (Theorem A: Basmajian's Identity, [3]) Let $M$ be a compact volume hyperbolic manifold with non-empty totally geodesic boundary and $V_{k}(r)$ the volume of the ball of radius $r$ in $\mathbb{H}^{k}$. Then

$$
\operatorname{Vol}(\partial M)=\sum_{l \in L_{M}} V_{n-1}\left(\log \left(\operatorname{coth} \frac{l}{2}\right)\right) .
$$

\subsection{Bridgeman-Kahn identity}

In the 2011 paper [13], Hyperbolic volume of n-manifolds with geodesic boundary and orthospectra, Bridgeman and Kahn obtained the following identity for the volume of the unit tangent bundle $T_{1}(M)$, again in terms of the ortholength spectrum $L_{M}$. 
Theorem 2.2. (Theorem B: Bridgeman-Kahn Identity, [13]) Let $M$ be a compact hyperbolic manifold with non-empty totally geodesic boundary, then

$$
\operatorname{Vol}\left(T_{1}(M)\right)=\sum_{l \in L_{M}} F_{n}(l)
$$

where $F_{n}: \mathbb{R}_{+} \rightarrow \mathbb{R}_{+}$is an explicitly described smooth monotonically decreasing function depending only on the dimension $n$.

We note that as $\operatorname{Vol}\left(T_{1}(M)\right)=\operatorname{Vol}(M) \cdot \operatorname{Vol}\left(\mathbb{S}^{n-1}\right)$, the above identity can also be thought of as an identity for the hyperbolic volume of the manifold $M$.

\subsection{The surface case of Theorems A and B}

Theorems A and B are particularly interesting in the case of hyperbolic surfaces as they give identities for deformation spaces of Riemann surfaces with boundary. They also relate in this context to the McShane and Luo-Tan identities which we describe in the next section. The Bridgeman-Kahn identity in fact arose from a generalization of a previous paper of the first named author 11] which provided an explicit formula for the function $F_{2}(l)$ in Theorem B in terms of the Roger's dilogarithm. We have:

Theorem 2.3. (Theorem $A^{\prime}$ : Basmajian identity for surfaces) Let $S$ be a hyperbolic surface with non-empty boundary $\partial S$. Then

$$
\operatorname{Length}(\partial S)=\sum_{l \in L_{S}} 2 \log \left(\operatorname{coth} \frac{l}{2}\right) .
$$

Theorem 2.4. (Theorem $B^{\prime}$ : Bridgeman identity) Let $S$ be a hyperbolic surface with non-empty boundary $\partial S$. Then

$$
\operatorname{Vol}\left(T_{1}(S)\right)=2 \pi \operatorname{Area}(S)=\sum_{l \in L_{S}} 4 . \mathcal{R}\left(\operatorname{sech}^{2} \frac{l}{2}\right)
$$

where $\mathcal{R}$ is the Rogers dilogarithm function.

The function $\mathcal{R}$ was introduced by Rogers in his 1907 paper [43]. This function arises in hyperbolic volume calculations; the imaginary part of $\mathcal{R}(z)$ is the volume of an ideal tetrahedron with vertices having cross-ratio $z$.

\subsection{Alternative derivations of the Bridgeman and Bridgeman-Kahn Identity}

In the paper [15], Calegari gives an alternate derivation of the Bridgeman identity. Also in [16], Calegari derived an orthospectrum identity for all dimensions 
$\geq 2$ which arose from a different decomposition of the unit tangent bundle, he showed in the surface case that it is equal to the Bridgeman identity. In a recent preprint 30 by Masai and McShane, it was shown that for higher dimensions, it is also equal to the Bridgeman-Kahn identity.

\section{Two Simple Spectra identities for hyperbolic surfaces}

Let $S$ be a finite area hyperbolic surface, we will consider various cases including when $S$ has cusps, totally geodesic boundary, cone singularities (with cone angles $\leq \pi$ ), and finally, when $S$ is a closed surface. We saw already in the previous section that when $S$ has non-empty totally geodesic boundary, we can define the collection of orthogeodesics $O_{S}$, which provided an index set for the Basmajian and Bridgeman identities, which are then expressed in terms of the ortholength spectrum $L_{S}$. This set can be extended in a natural way for surfaces which also have cusps or cone singularities. For the purposes of the next two classes of identities however, it is more useful to consider the smaller collection $\mathrm{SO}_{S}$ of simple orthogeodesics, that is, orthogeodesics which do not have self intersection, and also the collection $S G_{S}$ of simple closed geodesics on $S$. We will see that $S O_{S}$, together with collections of certain subsets of $S G_{S}$ consisting of one, two or three disjoint geodesics satisfying some topological criteria will be useful as index sets for the identities.

\section{1 (Generalized) McShane Identity}

Let $S_{g, n}$ denote a hyperbolic surface of genus $g$ with $n$ cusps. In his 1991 thesis, A remarkable identity for lengths of curves, McShane proved an identity for the lengths of simple closed geodesics on any once-punctured hyperbolic torus $S_{1,1}$ which he generalized later in 33 to more general cusped hyperbolic surfaces $S_{g, n}, n \geq 1$.

Theorem 3.1. (Theorem C: McShane Identity, [32], [33])

(1) If $S_{1,1}$ is a hyperbolic torus with one cusp, then

$$
\sum_{\gamma} \frac{1}{1+e^{l(\gamma)}}=\frac{1}{2}
$$

where the sum is over all simple closed geodesics $\gamma$ in $S_{1,1}$.

(2) If $S_{g, n}$ is a hyperbolic surface of genus $g$ with $n$ cusps, where $n \geq 1$, then

$$
\sum_{\gamma_{1}, \gamma_{2}} \frac{1}{1+\exp \left(\frac{l\left(\gamma_{1}\right)+l\left(\gamma_{2}\right)}{2}\right)}=\frac{1}{2}
$$


where the sum is over all unordered pairs of simple closed geodesics $\left\{\gamma_{1}, \gamma_{2}\right\}$ which bound together with a fixed cusp an embedded pair of pants in $S_{g, n}$. Here we adopt the convention that $\gamma_{1}$ or $\gamma_{2}$ may be one of the other cusps on $S_{g, n}$, considered as a (degenerate) geodesic of length 0.

The case of the punctured torus can be regarded as a special case of the surface $S_{g, n}$ where in the sum, $\gamma=\gamma_{1}=\gamma_{2}$ since any simple closed geodesic $\gamma$ on $S_{1,1}$ cuts it into a pair of pants.

In her 2005 thesis Simple geodesics and Weil-Petersson volumes of moduli spaces of bordered Riemann surfaces, see [36, 37, Mirzakhani gave a general version of the McShane identity for hyperbolic surfaces with geodesic boundaries and cusps, which was an important tool for her computation of the Weil-Petersson volumes of the moduli spaces. Independently, Ying Zhang in his 2004 thesis Hyperbolic cone surfaces, generalized Markoff Maps, Schottky groups and McShane's identity, see [51, 45, also gave a generalization of the McShane identity for surfaces, with (non-empty) boundary consisting of cusps, totally geodesic boundaries, or cone singularities with cone angle $\leq \pi$, with slightly different forms for the functions involved in the identity. We first state Mirzakhani's generalization and explain how to interprete the identity for cone surfaces later.

Theorem 3.2. (Theorem $C^{\prime}$ : Generalized McShane-Mirzakhani Identity, [36, 37]) Let $S$ be a finite area hyperbolic surface with geodesic boundary components $\beta_{1}, \ldots, \beta_{n}$, of length $L_{1}, \ldots, L_{n}$. Then

$$
\sum_{\left\{\gamma_{1}, \gamma_{2}\right\}} D\left(L_{1}, l\left(\gamma_{1}\right), l\left(\gamma_{2}\right)\right)+\sum_{i=2}^{n} \sum_{\gamma} R\left(L_{1}, L_{i}, l(\gamma)\right)=L_{1}
$$

where the first sum is over all unordered pairs of (interior) simple closed geodesics bounding a pair of pants with $\beta_{1}$, and the second sum is over simple closed geodesics bounding a pair of pants with $\beta_{1}, \beta_{i}$ and

$D(x, y, z)=2 \log \left(\frac{e^{\frac{x}{2}}+e^{\frac{y+z}{2}}}{e^{-\frac{x}{2}}+e^{\frac{y+z}{2}}}\right), R(x, y, z)=x-\log \left(\frac{\cosh \left(\frac{y}{2}\right)+\cosh \left(\frac{x+z}{2}\right)}{\cosh \left(\frac{y}{2}\right)+\cosh \left(\frac{x-z}{2}\right)}\right)$.

The case where some of the other boundaries are cusps but $\beta_{1}$ is a geodesic boundary can be deduced from the above, by considering cusps to be boundaries of length 0 , where again we adopt the convention that a cusp may be regarded as a geodesic of length 0 in the summands above. The case where $\beta_{1}$ is also a cusp, that is, $L_{1}=0$ is more interesting, in this case, the original McShane identities can be deduced from the above by taking the infinitesimal of the limit as $L_{1} \rightarrow 0$, or taking the formal derivative of the above identity with respect to $L_{1}$ and evaluating at $L_{1}=0$. More interestingly, a cone singularity of cone angle $\theta$ may be regarded as a boundary component with purely 
imaginary complex length $i \theta$, and the above identity is also valid if some of the boundary components are cone singularities of cone angles $\leq \pi$ as shown in [51, 45]. The restriction to cone angles $\leq \pi$ is necessary in the argument, this guarantees a convexity property and the existence of geodesic representatives for essential simple closed curves on the surface. For example, if $S$ is a surface of genus $g>1$ with one cone singularity $\Delta$ of cone angle $\theta$, then we have

$$
\sum_{\left\{\gamma_{1}, \gamma_{2}\right\}} D\left(i \theta, l\left(\gamma_{1}\right), l\left(\gamma_{2}\right)\right)=i \theta
$$

where $\left\{\gamma_{1}, \gamma_{2}\right\}$ are unordered pairs of simple closed geodesics bounding a pair of pants with $\Delta$. We note that each of the summands of the identity in Theorem $C^{\prime}$ is the measure of some subset $X_{i}$ of $\beta_{1}$ : the summands in the first sum of Theorem $C^{\prime}$ correspond to sets which are indexed by (a subset of) the simple orthogeodesics from $\beta_{1}$ to itself, the summands in the second sum correspond to sets which are indexed by simple orthogeodesics from $\beta_{1}$ to $\beta_{i}$ as we will see in the proof later.

\subsection{Luo-Tan identity}

The Basmajian, Bridgeman and McShane identities for surfaces were in general only valid for surfaces with boundary; in the first two cases, for surfaces with geodesic boundary, in the third case, to surfaces with at least some cusp or cone singularity. They do not extend to general closed surfaces without boundary. However, for the genus 2 hyperbolic surface $S_{2}$, by considering $S_{2} / h y p$ where hyp is the hyper-elliptic involution on $S_{2}$, one may lift the identity on the cone surface $S_{2} /$ hyp to obtain identities on the closed genus two surface $S_{2}$, see [35, 45]. This method however does not generalize to higher genus. In their 2011 preprint, A dilogarithm identity on moduli spaces of curves, F. Luo and the second author derived the following identity for closed hyperbolic surfaces.

Theorem 3.3. (Theorem D: Luo-Tan identity, [28]) Let $S$ be a closed hyperbolic surface. There exist functions $f$ and $g$ involving the dilogarithm of the lengths of the simple geodesic loops in a 3-holed sphere or 1-holed torus, such that

$$
\operatorname{Vol}\left(T_{1}(S)\right)=\sum_{P} f(P)+\sum_{T} g(T)
$$

where the first sum is over all properly embedded 3-holed spheres $P \subset S$ with geodesic boundary, the second sum is over all properly embedded 1-holed tori $T \subset S$ with geodesic boundary. 
The functions $f$ and $g$ are defined on the moduli spaces of simple hyperbolic surfaces (3-holed spheres and 1-holed tori) with geodesic boundary and given in terms of $\mathcal{R}$, the Rogers dilogarithm function as follows:

Suppose $P$ is a hyperbolic 3 -holed sphere with geodesic boundaries of lengths $l_{1}, l_{2}, l_{3}$. Let $m_{i}$ be the length of the shortest path from the $l_{i+1}$-th boundary to the $l_{i+2}$-th boundary $\left(l_{4}=l_{1}, l_{5}=l_{2}\right)$. Then

$$
f(P):=4 \sum_{i \neq j}\left[2 \mathcal{R}\left(\frac{1-x_{i}}{1-x_{i} y_{j}}\right)-2 \mathcal{R}\left(\frac{1-y_{j}}{1-x_{i} y_{j}}\right)-\mathcal{R}\left(y_{j}\right)-\mathcal{R}\left(\frac{\left(1-x_{i}\right)^{2} y_{j}}{\left(1-y_{j}\right)^{2} x_{i}}\right)\right]
$$

where $x_{i}=e^{-l_{i}}$ and $y_{i}=\tanh ^{2}\left(m_{i} / 2\right)$.

Suppose $T$ is a hyperbolic 1-holed torus with geodesic boundary. For any non-boundary parallel simple closed geodesic $A$ of length $a$ in $T$, let $m_{A}$ be the distance between $\partial T$ and $A$. Then $g(T):=$

$$
4 \pi^{2}+8 \sum_{A}\left[2 \mathcal{R}\left(\frac{1-x_{A}}{1-x_{A} y_{A}}\right)-2 \mathcal{R}\left(\frac{1-y_{A}}{1-x_{A} y_{A}}\right)-2 \mathcal{R}\left(y_{A}\right)-\mathcal{R}\left(\frac{\left(1-x_{A}\right)^{2} y_{A}}{\left(1-y_{A}\right)^{2} x_{A}}\right)\right]
$$

where $x_{A}=e^{-a}$ and $y_{A}=\tanh ^{2}\left(m_{A} / 2\right)$ and the sum is over all non-boundary parallel simple closed geodesics $A$ in $T$.

\subsection{Luo-Tan identity for surfaces with boundary and non-orientable surfaces}

The Luo-Tan identity also holds for surfaces $S$ with geodesic boundary and cusps, however, in this case, the functions $f$ and $g$ need to be modified when $P$ or $T$ share some boundary component with $S$, similar to the McShaneMirzakhani identity for surfaces with more than one boundary component, see 29]. In this case, the identity is trivial for the one-holed torus - however, one can obtain a meaningful identity involving the lengths of the simple closed geodesics in $T$ by a topological covering argument, using the identity for a four-holed sphere, see [19, 20]. For example, for a once-punctured hyperbolic torus $T$, we obtain

$$
\sum_{\gamma}\left[\mathcal{R}\left(\operatorname{sech}^{2}(l(\gamma) / 2)\right)+2\left(\mathcal{R}\left(\frac{1+e^{-l(\gamma)}}{2}\right)-\mathcal{R}\left(\frac{1-e^{-l(\gamma)}}{2}\right)\right)\right]=\frac{\pi^{2}}{2}
$$

where the sum extends over all simple closed geodesics $\gamma$ in $T$.

For non-orientable surfaces, one also obtain an analogous identity, in this case, the summands include terms coming from embedded simple non-orientable surfaces, namely, one holed Klein bottles and one-holed Möbius bands, see [29]. 


\section{Proofs of Theorems A and C - Boundary flow}

As remarked in the introduction, the proofs of all the results will be based on a decomposition of certain sets $X$ associated to $M$. In particular for Theorems A and $\mathrm{C}, X$ will be subsets of $T_{1}(M)$ associated to the boundary $\partial M$ and the proofs arise from consideration of the boundary flow on $X$.

\subsection{Proof of Basmajian Identity}

Let $T_{1}(M)$ be the unit tangent bundle of $M$ and $\pi: T_{1}(M) \rightarrow M$ be the projection map. For the Basmajian identity, we let $X$ be the set of unit tangent vectors $v$ whose basepoint are on $\partial M$, and which are perpendicular to $\partial M$, and point into the interior of $M$, that is,

$$
X=\left\{v \in T_{1}(M): \pi(v) \in \partial M, v \perp \partial M, v \text { points into } M\right\} .
$$

Clearly, $X$ identifies with $\partial M$ under $\pi$, and we define the measure $\mu$ on $X$ to be the pullback of Lebesgue measure on $\partial M$ under $\pi$. In particular, $\mu(X)=$ $\operatorname{Vol}(\partial M)$. We consider the unit speed geodesic $g_{v}$ starting at $p=\pi(v) \in \partial M$ obtained by exponentiating $v$. Thus $g_{v}$ is the geodesic arc obtained by flowing from $\pi(v)$ until you hit the boundary. To derive the Basmajian identity, we let

$$
Z=\left\{v \in X: \operatorname{Length}\left(g_{v}\right)=\infty\right\} .
$$

It follows from the fact that the limit set of $M$ is measure zero, that $Z$ has zero volume. For each of the remaining vectors, $g_{v}$ is a geodesic arc of finite length with endpoints on $\partial M$. We define an equivalence relation on these by defining $v \sim w$ if $g_{v}, g_{w}$ are homotopic rel. boundary in $M$. Then each oriented orthogeodesic $\alpha$ defines an equivalence class $E_{\alpha}$ given by

$$
E_{\alpha}=\left\{v \in X-Z \mid g_{v} \text { is homotopic rel boundary to } \alpha\right\} .
$$

Also, by lifting to the universal cover and using a tightening argument, we see that for every $v \in X \backslash Z, g_{v}$ is homotopic rel. boundary to a orthogeodesic $\alpha$, so $\left\{E_{\alpha}\right\}_{\alpha \in O_{M}}$ covers $X \backslash Z$. Furthermore if $\alpha \neq \beta$ then $E_{\alpha} \cap E_{\beta}=\emptyset$ as no two orthogeodesics are homotopic rel boundary. Thus we have the partition $X=Z \sqcup \bigsqcup_{\alpha} E_{\alpha}$ and the associated identity

$$
\operatorname{Vol}(X)=\operatorname{Vol}(\partial M)=\sum_{\alpha \in O_{M}} \operatorname{Vol}\left(E_{\alpha}\right)
$$

To calculate $\operatorname{Vol}\left(E_{\alpha}\right)$, we lift to the universal cover so that $\alpha$ lifts to a geodesic $\operatorname{arc} \tilde{\alpha}$ orthogonal to two boundary hyperplanes $P, Q$. As $\alpha$ is oriented, we assume $\tilde{\alpha}$ is oriented from $P$ to $Q$. Thus any $g_{v}$ homotopic rel boundary to $\alpha$ has a unique lift $\tilde{g}_{v}$ which is a geodesic arc perpendicular to $P$ going from $P$ 
to $Q$. Hence $\tilde{g}_{v}$ has basepoint in the orthogonal projection of $Q$ onto $P$. Thus the set $\pi\left(E_{\alpha}\right)$ lifts to a disk of radius $r(\alpha)$ given by orthogonal projection of $Q$ onto $P$. Let $r(\alpha), l(\alpha)$ be the two finite sides of a hyperbolic quadrilateral with one ideal vertex and finite angles $\pi / 2$ (see figure 2). By elementary hyperbolic geometry (see [5], for example), $\sinh (r(\alpha)) \cdot \sinh (l(\alpha))=1$, giving $r(\alpha)=\log (\operatorname{coth}(l(\alpha) / 2))$ and we obtain

$$
\operatorname{Vol}(\partial M)=\sum_{\alpha \in O_{M}} \operatorname{Vol}\left(E_{\alpha}\right)=\sum_{\alpha \in O_{M}} V_{n-1}(\log (\operatorname{coth}(l(\alpha) / 2))) .
$$

Remark: The identity generalizes to hyperbolic manifolds with cusps, as

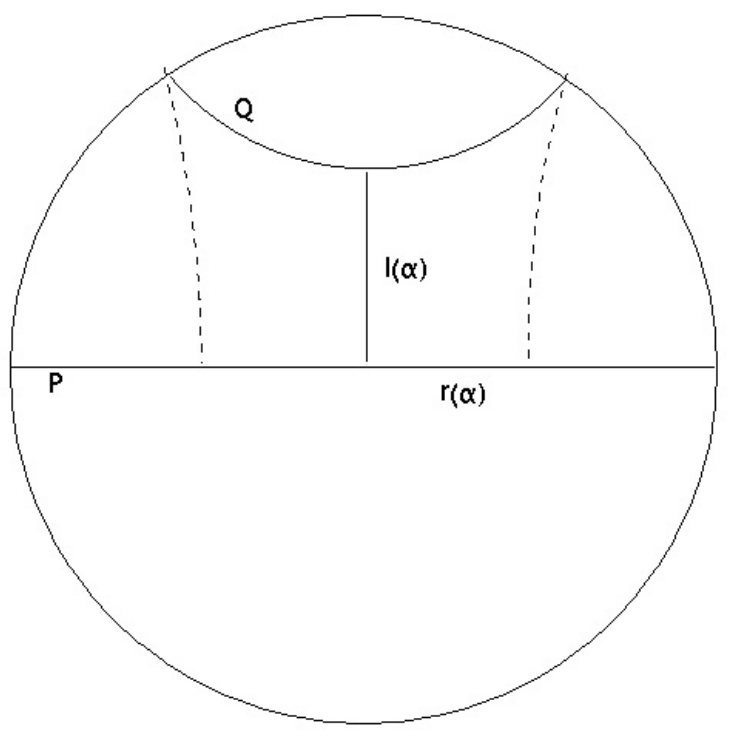

Figure 2. Orthogonal Projection onto a plane

long as the boundary contains some non-empty geodesic component. This is necessary to deduce that $\mu(Z)=0$. Othogeodesics ending in a cusp have infinite length and do not contribute to the summands of the identity.

\subsection{Proof of the Generalized McShane Identity}

We consider a hyperbolic surface $S$ with a finite number of geodesic boundary components, cusps and cone singularities (with cone angles $\leq \pi$ ).

For simplicity we first consider the case where $\partial S$ has only geodesic components $\beta_{1}, \ldots, \beta_{n}$ with lengths $L_{1}, \ldots, L_{n}$, the basic idea of the proof is the 
same for the more general case, we will explain later how to modify the proof if some of the $\beta_{i}$ 's are cusps or cone singularities of cone angle $\leq \pi$. We derive the identity based at $\beta_{1}$, as such, define

$$
X=\left\{v \in T_{1}(S): \pi(v) \in \beta_{1}, v \perp \beta_{1}, v \text { points into } S\right\} .
$$

Clearly, $\pi$ induces a bijection from $X$ to $\beta_{1}$, so $\mu(X)=\operatorname{Length}\left(\beta_{1}\right)=L_{1}$. Again, as in the proof of the Basmajian identity, we are going to consider the unit speed geodesic obtained by exponentiating $v \in X$, however, this time, we are going to stop when the geodesic hits itself, or the boundary $\partial S$. More precisely, let $G_{v}:[0, T] \rightarrow S$ be the geodesic arc obtained by exponentiating $v \in X$ such that $G_{v}$ is injective on $[0, T)$ and either $G_{v}(T)=G_{v}(s)$ for some $s \in[0, T)$, or $G_{v}(T) \in \partial S$. If $G_{v}[0, t]$ is defined and injective for all $t>0$, then $T=\infty$, that is $G_{v}$ is a simple geodesic arc of infinite length. A good analogy for the difference between $g_{v}$ in the Basmajian proof and $G_{v}$ in the McShane proof is that we should consider $g_{v}$ as a laser beam starting from $\partial M$ which is allowed to intersect itself any number of times, until it hits the boundary, whereas $G_{v}$ should be thought of as a wall, which terminates when it hits itself, or the boundary.

Now let $Z \subset X$ be the set of vectors for which $G_{v}$ has infinite length, that is, $T=\infty$. Again, by the same argument as before, $\mu(Z)=0$ since the limit set of $S$ has measure zero, and the endpoints of the lifts of $G_{v}$ must land on the limit set if $v \in Z$. However, if $\beta$ is a cusp, then we need a stronger result, namely, the Birman-Series result [6] that the set of simple geodesics on the surface has Hausdorff dimension 1, which implies that $\mu(Z)=0$. Similarly, if $\beta$ is a cone point, we require a generalization of the Birman-Series result, see [45]. We note that $Z$ has a rather complicated Cantor set structure, and McShane analysed this set carefully in 33. However, for the purposes of proving the identity, the structure of $Z$ is irrelevant, and one only really needs to know that $\mu(Z)=0$.

We now look at $G_{v}$ for $v \in X \backslash Z$, in this case $G_{v}$ is either a finite geodesic arc ending in a loop (a lasso), or a simple geodesic arc from $\beta_{1}$ to $\partial S$.

- If $G_{v}$ is a lasso, or a simple arc ending in $\beta_{1}$, then a regular neighborhood $N$ of $\beta_{1} \cup G_{v}$ in $S$ is topologically a pair of pants, where one of the boundary components is $\beta_{1}$. The other two boundary components can then be tightened to geodesics $\gamma_{1}, \gamma_{2}$ which are disjoint and which bound together with $\beta_{1}$ an embedded pair of pants in $S$ which contains $G_{v}$ (note that in the case where $S$ is a one-holed torus, then $\gamma_{1}=\gamma_{2}:=\gamma$, where $\gamma$ is a simple closed geodesic on $S$ disjoint from $G_{v}$, otherwise, $\gamma_{1}$ and $\gamma_{2}$ are distinct and disjoint).

- If $G_{v}$ is a simple arc from $\beta_{1}$ to $\beta_{i}$ where $i \neq 1$, then a regular neighborhood of $\beta_{1} \cup G_{v} \cup \beta_{i}$ is again a pair of pants, where $\beta_{1}$ and $\beta_{i}$ are two of the boundary components. The third boundary can again be tightened 
to a simple closed geodesic $\gamma$, and again $G_{v}$ is contained in the resulting pair of pants.

One can prove the assertions in the previous paragraph by a cut and paste argument as follows: Cut $S$ along $G_{v}$ to obtain a (not necessarily connected) convex hyperbolic surface $S_{c u t}$ with either two piece-wise geodesic boundaries (if $G_{v}$ is a lasso or simple arc from $\beta_{1}$ to itself), or one piece-wise geodesic boundary (if $G_{v}$ is a simple arc from $\beta_{1}$ to $\beta_{i}, i \neq 1$ ), and other geodesic boundaries. Note that if $S$ is a one-holed torus then $S_{c u t}$ is a cylinder whose core is a geodesic $\gamma$ disjoint from $\partial S_{c u t}$. Otherwise, in the first case, let $\gamma_{1}$ and $\gamma_{2}$ be the two disjoint geodesics which bound the convex core of $S_{c u t}$ which again are disjoint from $\partial S_{c u t}$. Regluing along $G_{v}$, we see that $\gamma_{1}$ and $\gamma_{2}$ bound together with $\beta_{1}$ a pair of pants in $S$ (basically the complement of the convex core of $S_{c u t}$ ) which contains $G_{v}$, as asserted. The same argument applies to the second case to obtain a pair of pants bounded by $\beta_{1}, \beta_{i}$ and a geodesic $\gamma$.

To recap, for every $v \in X \backslash Z, G_{v}$ is a geodesic arc contained in a unique pair of pants embedded in $S$ bounded by $\beta_{1}$ and a pair of geodesics $\gamma_{1}, \gamma_{2}$ (where one of $\gamma_{1}, \gamma_{2}$ may be a different boundary component $\beta_{i}$ of $S$ ). Let $\mathcal{P}$ be the set of all such pairs of pants embedded in $S$ (equivalently, all unordered pairs of geodesics $\left\{\gamma_{1}, \gamma_{2}\right\}$ in $S$ which bound a pair of pants with $\beta_{1}$ ), and for each $P \in \mathcal{P}$, we define

$$
X_{P}=\left\{v \in X \backslash Z: G_{v} \subset P\right\},
$$

then $X$ is the disjoint union of $Z$ and the $X_{P}$ 's, hence

$$
L_{1}=\sum_{P \in \mathcal{P}} \mu\left(X_{P}\right)
$$

To derive the formulae for $\mu\left(X_{P}\right)$ we consider a general pair of pants $P$ with boundary $a, b, c$ of lengths $x, y, z$. We consider perpendicular geodesics $\alpha_{p}$ from points $p$ on $a$. We let $p_{1}, p_{2}$ be the two points on $a$ such that $\alpha_{p_{i}}$ is a simple geodesic spiraling towards $b$ (one for each direction) and let $q_{1}, q_{2}$ be the two points on $a$ such that $\alpha_{q_{i}}$ is a simple geodesic spiraling towards $c$. Also assume that $p_{1}, q_{1}, q_{2}, p_{2}$ is the cyclic ordering of the points on $a$, which divide $a$ into the intervals $\left[p_{1}, q_{1}\right],\left[q_{1}, q_{2}\right],\left[q_{2}, p_{2}\right]$ and $\left[p_{2}, p_{1}\right]$ with disjoint interiors (see figure 3). Each interval contains a unique point $m$ such that $\alpha_{m}$ is a simple orthogeodesic from $a$ to $a, c, a$, and $b$ respectively. We see that for $p \in\left(p_{2}, p_{1}\right), \alpha_{p}$ is a simple geodesic from $a$ to $b$, similarly, for $p \in\left(q_{1}, q_{2}\right)$, $\alpha_{p}$ is a simple geodesic from $a$ to $c$. For $p \in\left(p_{1}, q_{1}\right) \cup\left(q_{2}, p_{2}\right), \alpha_{p}$ is either a simple geodesic from $a$ to itself or it has self-intersections. Furthermore, $L\left[p_{2}, p_{1}\right]$ is precisely the orthogonal projection of $b$ to $a$, similarly $L\left[q_{1}, q_{2}\right]$ is the orthogonal projection of $c$ to $a$, and $L\left[p_{1}, q_{1}\right]=L\left[q_{2}, p_{2}\right]$ by symmetry.

Now let $P \in \mathcal{P}$ where $a=\beta_{1}$. We have: 


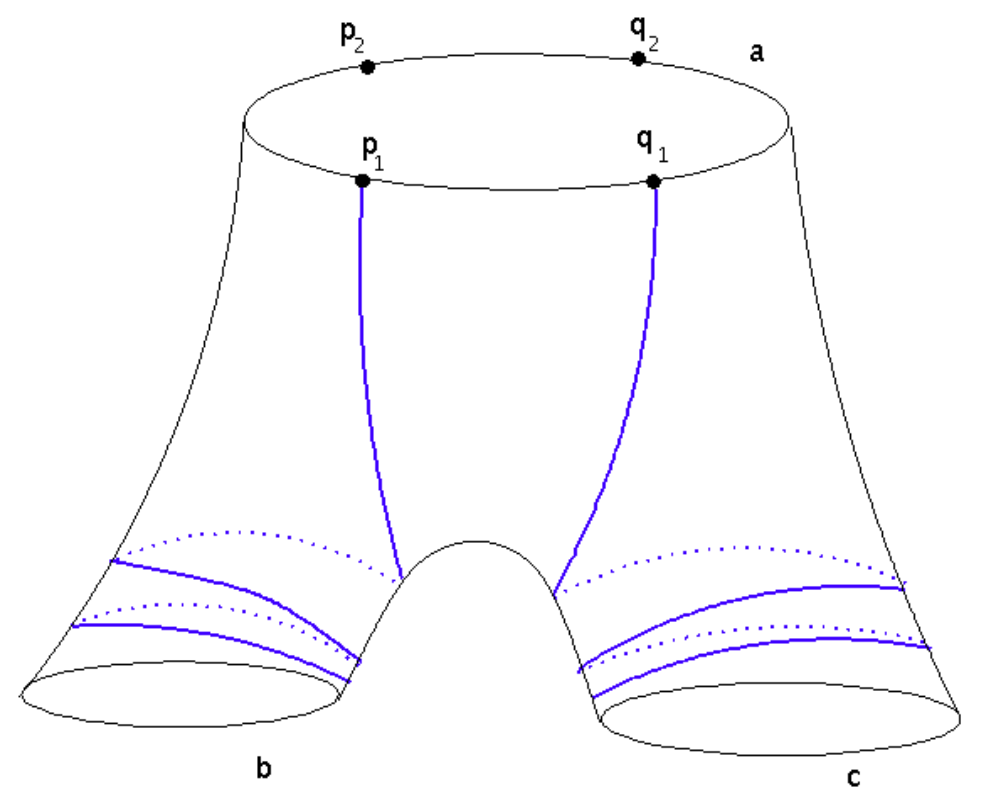

Figure 3. Pants $\mathrm{P}$ with spiraling geodesics

- If $b=\gamma_{1}$ and $c=\gamma_{2}$ are interior curves of $S$, then $G_{v} \subset X_{P}$ if and only if $\pi(v) \in\left(p_{1}, q_{1}\right) \cup\left(q_{2}, p_{2}\right)$. Then $\mu\left(X_{P}\right)=D(x, y, z)=2 L\left[p_{1}, q_{1}\right]$. By elementary hyperbolic geometry we have

$$
D(x, y, z)=2 \log \left(\frac{e^{\frac{x}{2}}+e^{\frac{y+z}{2}}}{e^{-\frac{x}{2}}+e^{\frac{y+z}{2}}}\right)
$$

- If say $b=\gamma$ is an interior geodesic and $c=\beta_{i}$ is a boundary geodesic of $S$, then $G_{v} \in X_{P}$ if and only if $\pi(v) \in\left(p_{1}, q_{1}\right) \cup\left(q_{1}, q_{2}\right) \cup\left(q_{2}, p_{2}\right)$. We have Length $\left[p_{2}, p_{1}\right]=\log (\operatorname{coth}(Z / 2))$ where $Z$ is the length of the perpendicular arc from $a$ to $b$. Applying the hyperbolic cosine rule we get

$$
R(x, y, z)=x-\log \left(\frac{\cosh \left(\frac{y}{2}\right)+\cosh \left(\frac{x+z}{2}\right)}{\cosh \left(\frac{y}{2}\right)+\cosh \left(\frac{x-z}{2}\right)}\right)
$$

The generalized McShane identity now follows by substitution. 


\subsection{Cusps and cone points}

If $\beta_{1}$ is a cusp, we can take a horocycle $C$ of length 1 about the cusp and remove the neighbourbood of the cusp bounded by $C$. We now take $X$ to be the set of vectors in $T_{1}(S)$ such that $\pi(v) \in C$ and $v$ is perpendicular to $C$ and pointing into $S$. Essentially the same analysis works to give a decomposition of $X$, with suitable modifications of the functions $D$ and $R$, which now only depend on two variables. In the tightening argument, we need the fact that the horocycle chosen is sufficiently small so that it is dijoint from all simple closed geodesics, choosing length one as we did works. If all other boundaries are cusps, then we recover the original McShane identity since in this case,

$$
D(y, z)=R(y, z)=\frac{1}{1+e^{\frac{y+z}{2}}} .
$$

If $\beta_{1}$ is a cone point of cone angle $\theta_{1}$, we decompose the set of tangent vectors based at the cone point again in essentially the same way. We note that in order for the suface obtained after cutting to be convex, the restriction that the cone angle $\leq \pi$ is necessary, we need this to perform the tightening argument. Similarly, we require all other cone angles to be $\leq \pi$ if we want every essential simple closed curve to be represented by a geodesic (or the double cover of a geodesic segment between two cone points of angle $\pi$ ).

Here it is useful to regard a cone point as an axis through the point perpendicular to the hyperbolic plane, and use the complex measure of length between two skew axes in $\mathbb{H}^{3}$. With this, the measure of the angle is purely imaginary. Similarly, other components $\beta_{i}$ which are cone angles should be regarded as axes perpendicular to the plane, and we recover exactly the same identity as that obtained by Mirzakhani, with the same functions, with the convention that cone points have purely imaginary lengths, see [45] for details.

\subsection{Index sets and the relation with Basmajian identity}

The set $X$ in the McShane identity is decomposed into the disjoint union of $Z$, a set of measure 0 and a countable union of disjoint open intervals $X_{\alpha}$, which from the previous observation is indexed by $\alpha \in S O_{S}\left(\beta_{1}\right)$, the set of simple orthogonal geodesics on $S$ with base point on $\beta_{1}$. Each such simple orthogeodesic gives rise to an interval in $\beta_{1}$, all of which are disjoint. The first sum consists of summands corresponding to the (two) intervals from the simple orthogeodesics from $\beta_{1}$ to itself contained in the pants $P$ where $\gamma_{1}$ and $\gamma_{2}$ are interior geodesics, a summand of the second sum consists of three intervals, the extra interval coming from the simple orthogeodesic contained in $P$ from $\beta_{1}$ to $\beta_{i}$.

If $\alpha \in S O_{S}\left(\beta_{1}\right)$ goes from $\beta_{1}$ to itself, then $\mu\left(X_{\alpha}\right)=L\left[p_{1}, q_{1}\right]=D(x, y, z) / 2$ (note that this length depends on the geometry of the pants $P$ and not just 
the length of $\alpha$ ) and if $\alpha$ is a simple orthogonal geodesic from $\beta_{1}$ to another

component $\beta_{i}$, then $\mu\left(X_{\alpha}\right)=2 \log \left(\operatorname{coth} \frac{l(\alpha)}{2}\right)$, the projection of $\beta_{i}$ to $\beta_{1}$ along $\alpha$.

The index set for the Basmajian identity is much larger, and strictly contains the index set for the McShane identity. In this sense, the Basmajian identity for surfaces, as restricted to $\beta_{1}$ is a refinement of the McShane identity: the terms corresponding to simple geodesics from $\beta_{1}$ to a different component $\beta_{i}$ are the same for both identities, however, in the McShane identity, each set $X_{\alpha}$ where $\alpha$ is a simple geodesic from $\beta_{1}$ to itself contains infinitely many terms in the Basmajian identities, as infinitely many non-simple orthogeodesics have the non-intersecting beginning part (the geodesic segment $G_{v}$ defined earlier) contained in the same pants $P$.

We note also that the index set for the McShane identity can be regarded as the set of all embedded pairs of pants in $S$ which contain $\beta_{1}$ as a boundary. These in turn split into two subsets, pairs of pants $P$ for which $\partial P \cap \partial S=\beta_{1}$ or $\partial P \cap \partial S=\beta_{1} \cup \beta_{i}$ for some $i \neq 1$. The first type gives the first sum, the second type the second sum in Theorem $B^{\prime}$. This point of view is useful as it generalizes to the Luo-Tan identity.

\section{Proofs of Theorems B and D: Interior Flow}

For the Bridgeman-Kahn and Luo-Tan identities, we consider $M$ a hyperbolic manifold and $T_{1}(M)$ its unit tangent bundle. We let $X=T_{1}(M)$ with $\mu$ the volume measure on $T_{1}(X)$. We then consider for each $v \in T_{1}(M)$ the geodesic obtained by flowing in both directions. We will show that the two identities described are obtained by considering the dynamical properties of this geodesic.

\subsection{Proof of the Bridgeman-Kahn identity}

Let $M$ be a hyperbolic manifold with totally geodesic boundary. We let $X=$ $T_{1}(M)$ and $\mu$ be the volume measure on $T_{1}(M)$. For each $v \in T_{1}(M)$ we let $g_{v}$ be the maximal geodesic arc tangent to $v$. To derive the Bridgeman-Kahn identity, we let $Z$ be the set of $v$ such that $g_{v}$ is not a proper geodesic arc (i.e. the flow does not hit the boundary in at least one direction). Once again, as the limit set of $M$ is measure zero, the set $Z$ satisfies $\mu(Z)=0$. For $v \notin Z$ we have that $g_{v}$ is a proper geodesic arc, and as in the Basmajian identity, we define an equivalence relation by $v \sim w$ if $g_{v}, g_{w}$ are homotopic rel boundary. Once again, each orthogeodesic $\alpha$ defines an equivalence class $E_{\alpha}$ 
and as before, they form a partition. Thus we have the associated identity

$$
\operatorname{Vol}\left(T_{1}(M)\right)=\sum_{\alpha \in O_{M}} \operatorname{Vol}\left(E_{\alpha}\right)
$$

To calculate $\operatorname{Vol}\left(E_{\alpha}\right)$, we lift $\alpha$ to the universal cover such that it is perpendicular to two boundary planes $P, Q$. By definition, any $v \in E_{\alpha}$ has $g_{v}$ homotopic rel boundary to $\alpha$. Thus $g_{v}$ has a unique lift to a geodesic $\tilde{g}_{v}$ which has endpoints on $P, Q$. Hence $E_{\alpha}$ also has a unique lift to $\tilde{E}_{\alpha}$ where

$$
\tilde{E}_{\alpha}=\left\{v \in T_{1}\left(\mathbb{H}^{n}\right) \mid \exists a \leq 0, b \geq 0 \text { such that } \tilde{g}_{v}(a) \in P, \tilde{g}_{v}(b) \in Q\right\} .
$$

The volume of this set only depends on $l(\alpha)=d(P, Q)$ and the dimension. Therefore we have

$$
\operatorname{Vol}\left(E_{\alpha}\right)=F_{n}(l(\alpha))
$$

for some function $F_{n}$ which gives the Bridgeman-Kahn identity. To derive a formula for $F_{n}$, we let $\Omega$ be the volume measure on the unit tangent bundle to the upper half space model for $\mathbb{H}^{n}$, invariant under $\operatorname{Isom}\left(\mathbb{H}^{n}\right)$. We let $G\left(\mathbb{H}^{n}\right)$ be the space of oriented geodesics in $\mathbb{H}^{n}$ and identify $G\left(\mathbb{H}^{n}\right)=\left(\mathbb{S}_{\infty}^{n-1} \times \mathbb{S}_{\infty}^{n-1} \backslash\right.$ Diagonal) by assigning to $g$ the pair of endpoints $(x, y)$. We have a natural fiber bundle $p: T_{1}\left(\mathbb{H}^{n}\right) \rightarrow G\left(\mathbb{H}^{n}\right)$ by letting $p(v)$ be the oriented geodesic tangent to $v$. We obtain a parametrization of $T_{1}\left(\mathbb{H}^{n}\right)$ by choosing a basepoint on each geodesic. We let $b \in \mathbb{H}^{n}$ and for each geodesic $g$, let $b_{g}$ be the nearest point of $g$ to $b$. Then to each $v \in T_{1}\left(\mathbb{H}^{n}\right)$ we assign the triple $(x, y, t) \in \mathbb{S}_{\infty}^{n-1} \times \mathbb{S}_{\infty}^{n-1} \times \mathbb{R}$ where $(x, y)=p(v)$ and $t$ is the signed hyperbolic distance along the geodesic $p(v)$ from $b_{p(v)}$ to $v$. In terms of this parametrization,

$$
d \Omega=\frac{2 d V_{x} d V_{y} d t}{|x-y|^{2 n-2}}
$$

where $d V_{x}=d x_{1} d x_{2} \ldots, d x_{n}$ and $|x-y|$ is the Euclidean distance between $x, y$ (see [38]). We choose planes $P, Q$ such that $d(P, Q)=l$ to be given by the planes intersecting the boundary in the circles of radius $1, e^{l}$ about the origin. We define

$$
E_{l}=\left\{v \in T_{1}\left(\mathbb{H}^{n}\right) \mid \exists a \leq 0, b \geq 0 \text { such that } \alpha_{v}(a) \in P, \alpha_{v}(b) \in Q\right\} .
$$

If $g=(x, y)$ is a geodesic intersecting planes both $P, Q$, we let $L(x, y, l)=$ $d(P \cap g, Q \cap g)$, the length between intersection points. Alternately we have $L(x, y, l)=\operatorname{Length}\left(p^{-1}(g) \cap E_{l}\right)$. Then integrating over $t$ we have

$$
F_{n}(l)=\int_{E_{l}} d \Omega=\int_{g \in p\left(E_{l}\right)}\left(\int_{p^{-1}(g) \cap E_{l}} d t\right) \frac{2 d V_{x} d V_{y}}{|x-y|^{2 n-2}}
$$


giving

$$
F_{n}(l)=\int_{|x|<1} \int_{|y|>e^{l}} \frac{2 L(x, y, l) d V_{x} d V_{y}}{|x-y|^{2 n-2}} .
$$

This integral formula can be simplified to obtain a closed form in even dimensions and can be reduced to an integral over the unit interval of a closed form in odd dimensions, in particular, when $n=2$, it takes on the explicit form given in the Bridgeman identity which we describe in the next subsection.

\subsection{Dilogarithm Identities}

We first describe the Rogers dilogarithm. We define the $k$ th polylogarithm function $L i_{k}$ to be the analytic function with Taylor series

$$
L i_{k}(z)=\sum_{n=1}^{\infty} \frac{z^{n}}{n^{k}} \quad \text { for }|z|<1 .
$$

Then we have

$$
L i_{0}(z)=\frac{z}{1-z} \quad L i_{1}(z)=-\log (1-z) .
$$

Also they satisfy the recursive formula

$$
L i_{k}^{\prime}(z)=\frac{L i_{k-1}(z)}{z} .
$$

The function $L i_{2}$ is the dilogarithm function and Rogers dilogarithm function is a normalization of it given by

$$
\mathcal{R}(z)=L i_{2}(z)+\frac{1}{2} \log |z| \log (1-z) .
$$

In the paper [11, the first author considered the surface case of the BridgemanKahn identity. We let $S$ be a finite area surface with totally geodesic boundary. Then boundary components of $S$ are either closed geodesics or bi-infinite geodesics (such as the case when $S$ is an ideal polygon). A boundary cusp of $S$ is a cusp on the boundary of $S$ bounded by two bi-infinite geodesics contained in the boundary of $S$. Let $N_{S}$ be the number of boundary cusps. Then we have the following generalized version

Theorem 5.1. (Bridgeman, [11]) Let $S$ be a finite area hyperbolic surface with totally geodesic boundary. Then

$$
\operatorname{Vol}\left(T_{1}(S)\right)=2 \pi \operatorname{Area}(S)=\sum_{l \in L_{S}} 4 \cdot \mathcal{R}\left(\operatorname{sech}^{2} \frac{l}{2}\right)+\frac{2 \pi^{2}}{3} N_{S} .
$$

where $\mathcal{R}$ is the Rogers dilogarithm function. 
In order to prove this, we once again take $X=T_{1}(S)$ and $\mu$ volume measure. We again define $Z$ to be the set of $v$ such $g_{v}$ is not a proper geodesic arc. Then if $v \notin Z, g_{v}$ is a proper geodesic arc and we define $v \sim w$ if $g_{v}, g_{w}$ are homotopic rel boundary. There are two cases, either $g_{v}$ is homotopic rel boundary to an orthogeodesic $\alpha$ or $g_{v}$ is homotopic to a neighborhood of a boundary cusp $c$. Thus we have equivalence classes $E_{\alpha}$ for each orthogeodesic and $E_{c}$ for each boundary cusp. Therefore

$$
\operatorname{Vol}\left(T_{1}(S)\right)=\sum_{\alpha} \operatorname{Vol}\left(E_{\alpha}\right)+\sum_{c_{i}} \operatorname{Vol}\left(E_{c_{i}}\right) .
$$

We have by definition that $\operatorname{Vol}\left(E_{\alpha}\right)=F_{2}(l(\alpha))$. Also, the sets $E_{c_{i}}$ are all isometric. Therefore

$$
\operatorname{Vol}\left(T_{1}(S)\right)=\sum_{l \in L_{S}} F_{2}(l)+N_{S} \operatorname{Vol}\left(E_{c}\right)
$$

As the above identity holds for $S$ an ideal triangle $T$ we have

$$
\operatorname{Vol}\left(T_{1}(T)\right)=2 \pi^{2}=3 \operatorname{Vol}\left(E_{c}\right) .
$$

Therefore

$$
\operatorname{Vol}\left(T_{1}(S)\right)=\sum_{l \in L_{S}} F_{2}(l)+\frac{2 \pi^{2}}{3} N_{S}
$$

The proof of the identity then follows by showing that $F_{2}(l)=4 \cdot \mathcal{R}\left(\operatorname{sech}^{2}\left(\frac{l}{2}\right)\right)$ where $\mathcal{R}$ is the Rogers dilogarithm (in [11] the index was over all unoriented orthogeodesics so the constant there was 8). In [11] this is done by directly computing the integral in the formula 5.1 for $F_{2}$. We now describe an alternate approach that avoids this computation.

\subsection{Finite identities}

The surface identity is only a finite identity when $S$ is an ideal n-gon. In this case we have a finite orthospectrum $l_{1}, \ldots, l_{k}$. We then have

$$
2 \pi^{2}(n-2)=\sum_{i} F_{2}\left(l_{i}\right)+\frac{2 \pi^{2} n}{3} .
$$

We rewrite this as

$$
\sum_{i} F_{2}\left(l_{i}\right)=\frac{4 \pi^{2}(n-3)}{3}
$$


We let $R$ be the function defined such that $F_{2}(l)=8 \cdot R\left(\operatorname{sech}^{2}\left(\frac{l}{2}\right)\right)$. Then $R$ satisfies

$$
\sum_{i} R\left(\operatorname{sech}^{2}\left(\frac{l_{i}}{2}\right)\right)=\frac{(n-3) \pi^{2}}{6}
$$

If the ideal polygon $S$ has cyclically ordered vertices $x_{i}$, then the $i$ th side can be identified with the geodesic with endpoints $x_{i}, x_{i+1}$. Then an orthogeodesic is the perpendicular between geodesic $x_{i}, x_{i+1}$ and geodesic $x_{j}, x_{j+1}$ where $|i-j| \geq 2$ which we label $\alpha_{i j}$ of length $l_{i j}$. We define the cross ratio of four points by

$$
[a, b ; c, d]=\frac{(a-b)(d-c)}{(a-c)(d-b)}
$$

Then a simple calculation shows that

$$
\left[x_{i}, x_{i+1} ; x_{j}, x_{j+1}\right]=\left[1,-1,-e^{l_{i j}}, e^{l_{i j}}\right]=\frac{(1+1)\left(e^{l_{i j}}+e^{l_{i j}}\right)}{\left(1+e^{l_{i j}}\right)\left(1+e^{l_{i j}}\right)}=\operatorname{sech}^{2}\left(l_{i j} / 2\right)
$$

Therefore we can rewrite the identity in the finite case as

$$
\sum_{|i-j| \geq 2} R\left(\left[x_{i}, x_{i+1} ; x_{j}, x_{j+1}\right]\right)=\frac{(n-3) \pi^{2}}{6} .
$$

Ideal Quadrilaterals and Euler's identity: For $n=4, S$ is an ideal quadrilateral with two ortholengths $l_{1}, l_{2}$ and vertices $x_{1}, x_{2}, x_{3}, x_{4}$. Thus as $\left[x_{1}, x_{2} ; x_{3}, x_{4}\right]=1-\left[x_{3}, x_{4} ; x_{1}, x_{2}\right]$, for any $0<x<1$ we have

$$
R(x)+R(1-x)=\frac{\pi^{2}}{6} .
$$

This identity was proved for the dilogarithm function by Euler (see Lewin's book 27] for details). Also by symmetry $R(1 / 2)=\pi^{2} / 12$ and similarly $R(0)=$ $0, R(1)=\pi^{2} / 6$.

Ideal Pentagons and Abel's identity: If $S$ is a ideal pentagon then there are 5 orthogeodesics. We send three of the vertices to $0,1, \infty$ and the other two to $u, v$ with $0<u<v<1$. Then the cross ratios in terms of $u, v$ are

$$
u, \quad 1-v, \quad \frac{v-u}{v}, \quad \frac{v-u}{1-u}, \quad \frac{u(1-v)}{v(1-u)}
$$

Putting into the equation we obtain the following equation.

$$
R(u)+R(1-v)+R\left(\frac{v-u}{v}\right)+R\left(\frac{v-u}{1-u}\right)+R\left(\frac{u(1-v)}{v(1-u)}\right)=\frac{\pi^{2}}{3} .
$$


Letting $x=u / v, y=v$, we get

$$
R(x y)+R(1-y)+R(1-x)+R\left(\frac{y(1-x)}{1-x y}\right)+R\left(\frac{x(1-y)}{1-x y}\right)=\frac{\pi^{2}}{3} .
$$

Now by applying Euler's identity for $x, y$, we obtain Abel's pentagon identity

$$
R(x)+R(y)=R(x y)+R\left(\frac{y(1-x)}{1-x y}\right)+R\left(\frac{x(1-y)}{1-x y}\right) .
$$

To show $R=\mathcal{R}$ we use the observation of Calegari in [15], that by a result of Dupont (see [17]), the Rogers dilogarithm is characterized by satisfying the Euler and Abel identities, and therefore $R=\mathcal{R}$.

\subsection{Proof of the Luo-Tan identity}

We let $S$ be a closed hyperbolic surface. We note that as $\partial S=\emptyset$, the Basmajian and Bridgeman identities do not make sense as there are no orthogeodesics. Similarly, it is not clear how to extend the McShane identity to this case as there is no starting point, i.e., no boundary component or horocycle to decompose. Furthermore, since the generalization of the McShane identity to cone surfaces has a restriction that all cone angles are $\leq \pi$, we cannot deform a cone singularity to a smooth point to obtain an identity.

However, one can combine two key ideas from the Bridgeman-Kahn and the McShane proofs to obtain an identity, which is what Luo and the second author did. The key idea from the Bridgeman-Kahn identity is to start from any point and any direction, that is, to define $X=T_{1}(S)$ with $\mu$ the volume measure. The key idea we use from the proof of the McShane identity is that instead of flowing in both directions indefinitely, as in the Bridgeman identity, we flow until we get intersection points. In this way, for a generic vector $v \in T_{1}(S)$, we construct a geodesic graph $G_{v}$ with Euler characteristic -1 and use this to obtain a decomposition of $X$. From this decomposition we calculate the measures of the components to obtain the Luo-Tan identity.

More precisely, given $v \in T_{1}(S)$, consider the unit speed geodesic rays $\gamma_{v}^{+}(t)$ and $\gamma_{v}^{-}(t)(t \geq 0)$ determined by exponentiating $\pm v$. If the vector $v$ is generic, then both rays will self intersect transversely by the ergodicity of the geodesic flow, otherwise, we have $v \in Z \subset X$ where $\mu(Z)=0$.

Each $v \in X \backslash Z$ will determine a canonical graph $G_{v}$ as follows (see figure 4). Consider the path $A_{t}=\gamma_{v}^{-}([0, t]) \cup \gamma_{v}^{+}([0, t])$ for $t>0$ obtained by letting the geodesic rays $g_{v}^{-}$and $g_{v}^{+}$grow at equal speed from time 0 to $t$. Let $t_{+}>0$ be the smallest positive number so that $A_{t_{+}}$is not a simple arc, without loss of generality, we may assume that $\gamma_{v}^{+}\left(t_{+}\right) \neq \gamma_{v}^{-}\left(t_{+}\right)$by ignoring a set of measure zero (i.e. putting it into $Z)$. Say $\gamma_{v}^{+}\left(t_{+}\right) \in \gamma_{v}^{-}\left(\left[0, t_{+}\right]\right) \cup \gamma_{v}^{+}\left(\left[0, t_{+}\right)\right)$. Next, let $t_{-}>t_{+}$be the next smallest time so that $\left.\gamma_{v}^{-}\left(t_{-}\right) \in \gamma_{v}^{-}\left(\left[0, t_{-}\right)\right) \cup \gamma_{v}^{+}\left[0, t_{+}\right]\right)$. 


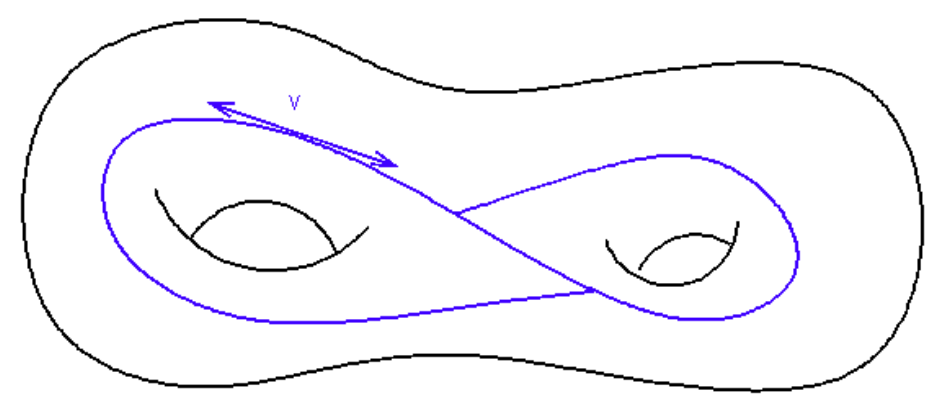

Figure 4. Graph $G_{v}$

Definition 5.2. The union $\gamma_{v}^{-}\left(\left[0, t_{-}\right]\right) \cup \gamma_{v}^{+}\left(\left[0, t_{+}\right]\right)$is the graph $G_{v}$ associated to $v$.

From the definition, $G_{v}$ has Euler characteristic is -1 . We call an embedded pair of pants (three hole sphere) or one-hole torus with geodesic boundary in $S$ a simple geometric embedded subsurface. The following result allows us to decompose $X \backslash Z$ into subsets indexed by the set of simple geometric embedded subsurfaces.

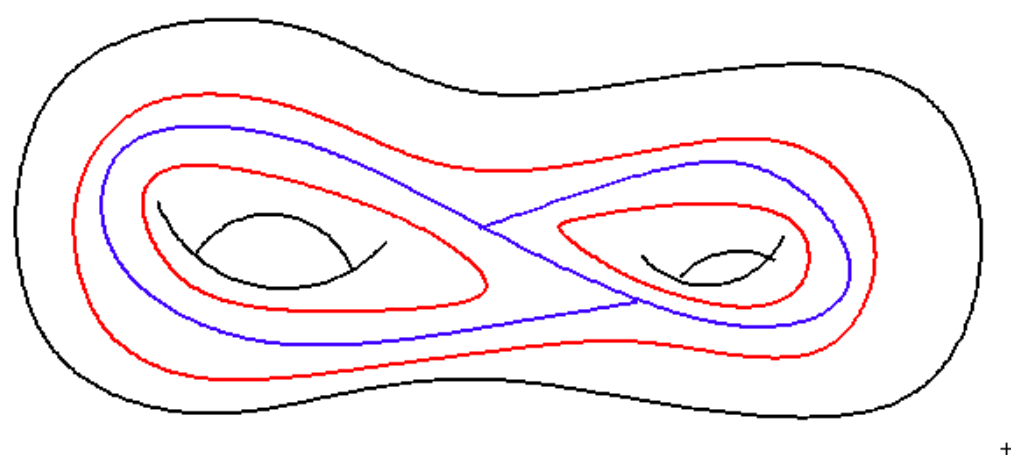

Figure 5. Subsurface $\Sigma_{v}$, with $G_{v} \subset \Sigma_{v}$

Proposition 5.3. (Proposition 3.1 of [28]) The graph $G_{v}$ is contained in a unique simple geometric embedded subsurface $\Sigma_{v} \subset S$.

Proof. Cutting the surface $S$ open along $G_{v}$, we obtain a surface whose metric completion $\hat{S}$ is a compact hyperbolic surface with convex boundary. The 
boundary of $\hat{S}$ consists of simple piecewise geodesic loops (corresponding to $G_{v}$ ), each boundary has at least one corner. If $\hat{\gamma}$ is a simple piecewise geodesic loop in $\partial \hat{S}$, it is freely homotopic to a simple closed geodesic $\gamma$ in $\hat{S}$ which is a component of the boundary of core $(\hat{S})$, the convex core of $\hat{S}$. Furthermore $\hat{\gamma}$ and $\gamma$ are disjoint by convexity. Therefore, $\hat{\gamma}$ and $\gamma$ bound a convex annulus exterior to $\operatorname{core}(\hat{S})$ and $G_{v}$ is disjoint from core $(\hat{S})$. The simple geometric subsurface $\Sigma_{v}$ containing $G_{v}$ is the union of these convex annuli bounded by $\hat{\gamma}$ and $\gamma$ (see figure 5). The Euler characteristic of $\Sigma_{v}$ is -1 by the construction. Furthermore, the surface $\Sigma_{v}$ is unique. Indeed, if $\Sigma^{\prime} \neq \Sigma \subset S$ is a simple geometric subsurface so that $G_{v} \subset \Sigma^{\prime}$, then $\Sigma^{\prime}$ has a boundary component say $\beta$ which intersects one of the boundaries $\gamma$ of $\Sigma$ transversely (we use here in an essential way the fact that the surfaces $\Sigma$ and $\Sigma^{\prime}$ are simple). Therefore, $\beta$ must intersect the other boundary $\hat{\gamma}$ of the convex annulus described earlier, otherwise we have a hyperbolic bigon, a contradiction. Hence it intersects $G_{v}$ which contradicts $G_{v} \subset \Sigma^{\prime}$.

The above discussion gives a decomposition of $T_{1}(S) \backslash Z$ indexed by these simple subsurfaces, namely, for any simple subsurface $\Sigma \subset S$, define

$$
X_{\Sigma}:=\left\{v \in X: G_{v} \subset \Sigma\right\} .
$$

Then $\mu(X)=\mu\left(T_{1}(S)\right)=\sum_{P} \mu\left(X_{P}\right)+\sum_{T} \mu\left(X_{T}\right)$ where the first sum is over all simple geometric embedded pairs of pants and the second sum over all simple geometric embedded one-hole tori. It remains to calculate for a simple hyperbolic surface $\Sigma=P$ or $T$ the volume of the set of all unit tangent vectors $v \in T_{1}(\Sigma)$ so that $G_{v}$ is strictly contained in $\Sigma$, that is, $G_{v}$ is a spine for $\Sigma$, this will give us the functions $f$ and $g$ in Theorem $D$.

\subsection{Computing the measures of $X_{P}$ and $X_{T}$}

By definition, we have $f(P)=\mu\left(X_{P}\right)$ and $g(T)=\mu\left(X_{T}\right)$. It is complicated to compute the measures directly, it turns out that it is easier to compute $\mu\left(X_{P}\right)$ and $\mu\left(X_{T}\right)$ by calculating the measure of the complementary set in $T_{1}(\Sigma)$ instead. The idea is that the vectors $v \in T_{1}(P)$ in the complementary set can be divided into a small number of disjoint types which can be described quite easily geometrically, hence the complementary set decomposes into a finite number of disjoint subsets whose measures can be computed in a similar way to the computation for the Bridgeman identity.

Suppose $P$ is a hyperbolic 3 -holed sphere, and $i \in\{1,2,3\}$ taken mod 3 . Denote the boundary geodesics of $P$ by $L_{i}$, the simple orthogeodesics from $L_{i+1}$ to $L_{i+2}$ by $M_{i}$ and the simple orthogeodesic from $L_{i}$ to itself by $N_{i}$, see figure 6(a). Denote the lengths of the boundary geodesics and the orthogeodesics by the corresponding lower case letters, that is $l_{i}, m_{i}$ and $n_{i}$ respectively. 


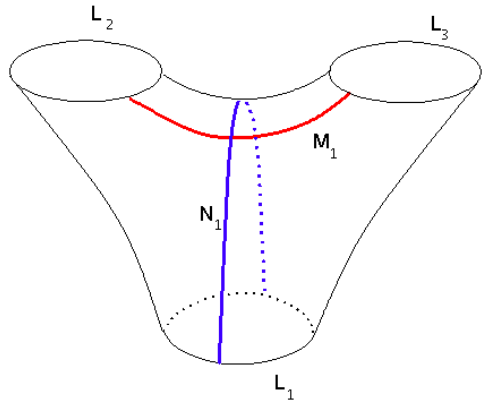

(a)

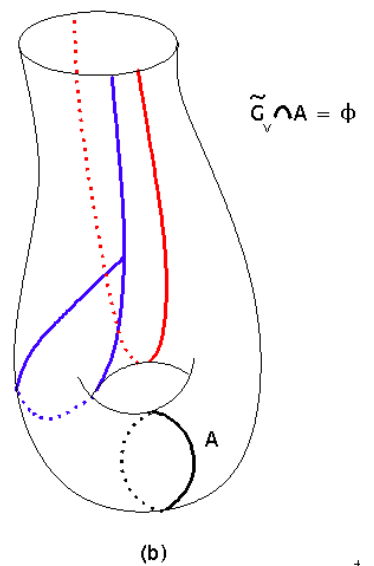

(b)

Figure 6. 3-holed spheres and 1-holed tori

For $v \in T_{1}(P)$ we define $\gamma_{v}$ to be the maximal geodesic arc in $P$ tangent to $v$. We modify the definition of $G_{v}$ appropriately, to take into account the fact that $\partial P \neq \emptyset$, that is,

$$
\hat{G}_{v}=\gamma_{v}^{-}\left(\left[0, t_{-}\right]\right) \cup \gamma_{v}^{+}\left(\left[0, t_{+}\right]\right)
$$

as before but the times $t_{+}$and $t_{-}$may also occur when the geodesic hits $\partial P$ in which case we stop generating the geodesic in that direction.

From the definitions, it is clear that $\hat{G}_{v} \subset \gamma_{v}$, and $v \in X_{P}$ if and only if $\hat{G}_{v} \cap \partial P=\emptyset$. In particular, if $v \in T_{1}(P) \backslash X_{P}$, and $v \notin Z$, then $\hat{G}_{v}$ is either simple, with both endpoints on $\partial P$, or has one endpoint on $L_{i}$ and the other end is a loop freely homotopic to $L_{j}, j \neq i$, we call this a lasso around $L_{j}$ based at $L_{i}$ (note that the loop cannot be homotopic to $L_{i}$ ). The following gives a decomposition of $T_{1}(P) \backslash X_{P}$ into finitely many types:

- Define $H\left(M_{i}\right)=\left\{v \in T_{1}(P) \mid \gamma_{v} \sim M_{1}\right.$ rel. boundary $\}$. If $v \in H\left(M_{i}\right)$, then $\gamma_{v}$ is simple, $\hat{G}_{v}=\gamma_{v}$, and $v \notin X_{P}$. The measure of these sets, computed by Bridgeman in [11, depend only on $m_{i}$ and is given by $\mu\left(H\left(M_{1}\right)\right)=8 . \mathcal{R}\left(\operatorname{sech}^{2} \frac{m_{i}}{2}\right)$.

- Define $H\left(N_{i}\right)=\left\{v \in T_{1}(P) \mid \gamma_{v} \sim N_{1}\right.$ rel. boundary $\}$. If $v \in H\left(N_{i}\right)$, then $\gamma_{v}$ intersects $M_{i}$ exactly once, the point of intersection divides $\gamma_{v}$ into two components $\gamma_{v}^{+}$and $\gamma_{v}^{-}$. This case is more complicated as $\gamma_{v}$ may have arbitrarily many self-intersections, however, $\gamma_{v}^{+}$and $\gamma_{v}^{-}$ are both simple. This can be seen by cutting $P$ along $M_{i}$ to obtain a convex cylinder bounded on one side by $L_{i}$ and the other by a piecewise geodesic boundary. Then both $\gamma_{v}^{+}$and $\gamma_{v}^{-}$are geodesic arcs from one boundary of the cylinder to the other, so must be simple. In particular, 
in the construction of $\hat{G}_{v}$, we see that in this case, $\hat{G}_{v}$ is either a simple geodesic arc from $L_{i}$ to itself, or it must be a lasso based at $L_{i}$. That is, for all such $v, \hat{G}_{v}$ intersects $L_{i}$ so $v \notin X_{P}$. Again, by Bridgeman the measure is $\mu\left(H\left(N_{i}\right)\right)=8 . \mathcal{R}\left(\operatorname{sech}^{2} \frac{n_{i}}{2}\right)$. Note that these sets are disjoint from those in the first case.

- The remaining case is when $\hat{G}_{v}$ is a lasso, but does not come from case two above, we call these true lassos. For $\{i, j, k\}=\{1,2,3\}$ distinct, let $W\left(L_{i}, M_{j}\right)=\left\{v \in T_{1}(P) \mid \hat{G}_{v}\right.$ is a true lasso around $L_{i}$ based at $\left.L_{k}\right\}$.

Consider say that $\hat{G}_{v}$ is a lasso based at the point $q$ on $L_{1}$ with (positive) loop around $L_{2}$, but such that $\gamma_{v}$ is not homotopic relative to the boundary to $N_{1}$. It is convenient to understand the set of $v$ generating such $\hat{G}_{v}$ in the universal cover, $\tilde{P} \subset \mathbb{H}^{2}$ (we use the upper half space model). We work with the following setting:

Consider a fundamental domain for $P$ in $\mathbb{H}^{2}$ consisting of two adjacent right angled hexagons $H$ and $H^{\prime}$ such that $H$ is bounded by $\tilde{L}_{1}$, $\tilde{L}_{2}$ and $\tilde{L}_{3}$ and $H^{\prime}$ is bounded by $\tilde{L}_{2}, \tilde{L}_{1}^{\prime}$ and $\tilde{L}_{3}$, see figure 7 Given $x, y \in \mathbb{H}^{2} \cup \partial \mathbb{H}^{2}, x \neq y$, let $G[x, y]$ denote the geodesic in $\mathbb{H}^{2}$ from $x$ to $y$. Normalize so that $\tilde{L}_{1}=G[c, d], \tilde{L}_{2}=G[\infty, 0]$, and $\tilde{L}_{3}=G[e, f]$, where $e, f, c, d \in \mathbb{R}$ satisfy $0<e<f<c<d$. Further normalize so that $\tilde{L}_{1}^{\prime}=G[1, a]$ where $1<a<e$. By elementary calculations, we have $c=e^{l_{2}}, d=e^{l_{2}} \operatorname{coth}^{2}\left(m_{3} / 2\right)$.

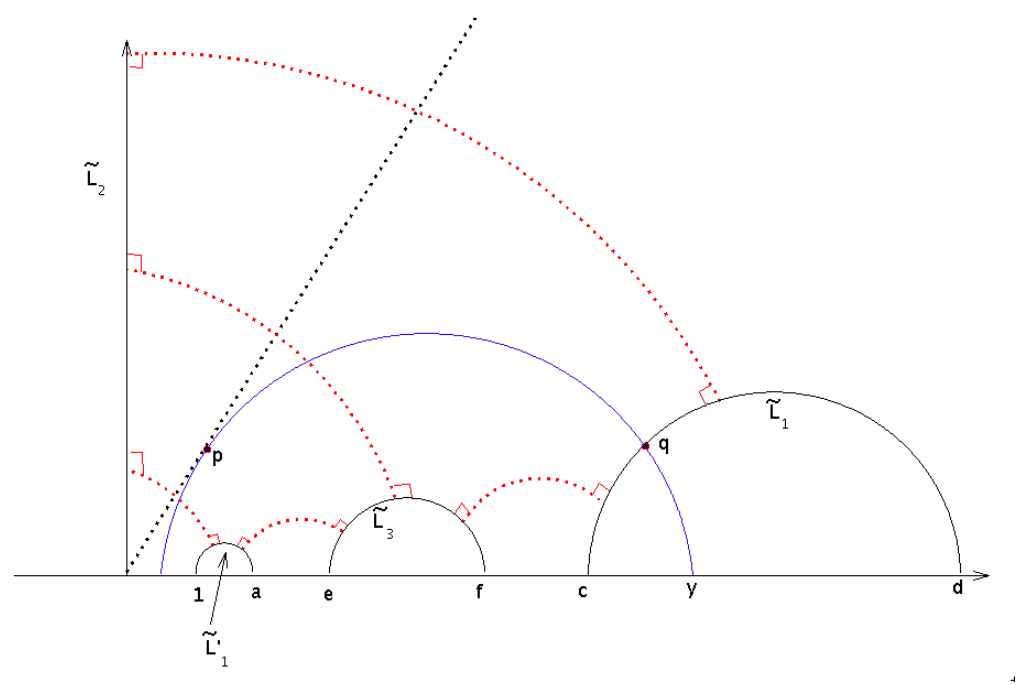

Figure 7. Universal Cover of $P$ in $\mathbb{H}^{2}$ 
We can choose a lift of $\hat{G}_{v}$ so that the end point $q$ lies on $\tilde{L}_{1}=G[c, d]$. Let $G[y, x], y, x \in \mathbb{R}^{2}$ be the complete geodesic in $\mathbb{H}^{2}$ containing this lift, in particular, by construction $y \in(c, d)$. We claim that there is a unique lift so that $0<x<1$. The cases $x=0$ are $x=1$ are important limiting cases. When $x=0, \hat{G}_{v}$ is a simple geodesic of infinite length which spirals around $L_{2}$ in the positive direction, when $x<0, \hat{G}_{v}$ is homotopic to $M_{3}$, so is a simple finite geodesic arc from $L_{1}$ to $L_{2}$. When $x$ is small and positive, $\hat{G}_{v}$ is a lasso with positive loop around $L_{2}$. When $x=1, \gamma_{v}$ spirals around $L_{1}$ with infinite number of self intersections, and $\hat{G}_{v}$ is still a lasso from $L_{1}$ with positive loop around $L_{2}$. As $x$ ranges between 1 and $a, \gamma_{v}$ is homotopic to $N_{1}$ and $\hat{G}_{v}$ ranges from being a lasso with positive loop around $L_{2}$ to being simple and then a lasso with negative loop around $L_{3}$. In particular, if $\hat{G}_{v}$ is a lasso with end point at $L_{1}$ and positive loop around $L_{2}$, but $\gamma_{v}$ is not homotopic to $N_{1}$ we have a unique lift so $0<x<1$ and $c<y<d$. However, not all tangent vectors on $T_{1}(G[y, x])$ generate $\hat{G}_{v}$. By the construction of $\hat{G}_{v}$, the midpoint of the loop, $p$ of $\hat{G}_{v}$ is a critical point, all vectors which project to the longer part of $\hat{G}_{v}$ will generate $\hat{G}_{v}$, those which project to the shorter part (consisting of half of the loop) will generate a different $\hat{G}_{v}$, by construction. Also, the loop of $\hat{G}_{v}$ bounds with $L_{2}$ a convex cylinder in $P$ so $p$ is the point on the loop which is closest to $L_{2}$. Hence, $p$ is the point on $G[y, x]$ such that the ray $0 p$ is tangent to $G[y, x]$. To calculate the volume, we need to integrate the length of the geodesic from $q$ to $p$ over the set of all geodesics in $\mathbb{H}^{2}$ with endpoints $x, y$ where $0<x<1$ and $c<y<d$, with respect to the Liouville measure on the space of geodesics. Since both forward and backward vectors on this set generate $\hat{G}_{v}$, we multiply the result by 2 , that is we want twice the volume of the set $\Omega$ where

$$
\Omega=\left\{v \in T_{1}\left(\mathbb{H}^{2}\right) \mid v \in T_{1}(G[q, p]), 0<x<1, c<y<d\right\} .
$$

The computation of $\operatorname{Vol}(\Omega)$ is elementary but somewhat messy, the final integral which needs to be computed is given by the following:

Proposition 5.4. (Prop 4.1 of [28]) The volume of $\Omega$ is given by

$$
\int_{0}^{1}\left(\int_{c}^{d} \frac{\ln \left|\frac{y(x-c)(x-d)}{x(y-c)(y-d)}\right|}{(y-x)^{2}} d y\right) d x
$$

Note that the above integral only depends on $c$ and $d$ which are given by $c=e^{l_{2}}, d=e^{l_{2}} \operatorname{coth}^{2}\left(m_{3} / 2\right)$. More generally, define the Lasso function $L a(l, m)$ to be the above integral where $c=e^{l}, d=e^{l} \operatorname{coth}^{2}(m / 2)$. We have: 
Proposition 5.5. (Prop 4.6 of [28]) The lasso function $L a(l, m)$ is given by

$$
L a(l, m)=2\left(\mathcal{R}(y)-\mathcal{R}\left(\frac{1-x}{1-x y}\right)+\mathcal{R}\left(\frac{1-y}{1-x y}\right)\right)
$$

where $x=e^{-l}$ and $y=\tanh ^{2}(m / 2), \mathcal{R}(z)$ is the Roger's dilogarithm.

As remarked earlier, we need to take twice the volume of $\Omega$ to obtain the volume of the set of vectors generating true lassos from $L_{1}$ with a positive loop around $L_{2}$, the volume for those with a negative loop around $L_{2}$ is the same by symmetry, hence we have:

$$
\operatorname{Vol}\left(W\left(L_{i}, M_{j}\right)\right)=4 L a\left(l_{i}, m_{j}\right) .
$$

Now $\mu\left(X_{P}\right)$ can be computed by subtracting away from $\mu\left(T_{1}(P)\right)$ the measures of the sets $H\left(M_{i}\right), H\left(N_{i}\right), i=1,2,3$ and $W\left(L_{i}, M_{j}\right), i \neq j$. To obtain the expression in Theorem $\mathrm{D}$, we use some of the pentagon relations satisfied by the Roger's dilogarithm function, for details, see 28.

The computation of $\mu\left(X_{T}\right)$ for an embedded one-holed torus is similar, with an extra observation. Again, $v \notin X_{T}$ if $\hat{G}_{v} \cap \partial T \neq \emptyset$, ( $\partial T$ has only one component). In this case $\hat{G}_{v}$ is either a simple geodesic arc from $\partial T$ to itself, or is a lasso based at $\partial T$ with a loop homotopic to an essential, non boundary parallel simple closed geodesic. In either case, $\hat{G}_{v}$ is disjoint from a unique simple closed geodesic $A \subset T$, see figure $6 \mathrm{~b}$. Cutting along $A$ produces a pair of pants $P_{A}$, and from the previous calculations, we can calculate the set of all $v \in T_{1}\left(P_{A}\right)$ such that $\hat{G}_{v}$ intersects $\partial T$ but not the other two boundary components of $P_{A}$. Summing up the measures over all possible simple closed geodesics $A$ we obtain the measure of the complement of $X_{T}$ in $T_{1}(T)$. Again, by manipulating the expressions using the identities for $\mathcal{R}$, we obtain the expression in Theorem D for $g(T)$, see [28] for details.

\section{Moments of hitting function}

We consider all four identities and their associated measure space $(X, \mu)$. Associated to this we have a hitting function $L: X \rightarrow \mathbb{R}_{+}$where $L(x)$ is the length of the geodesic arc associated to $x$. The function $L$ is measurable and we can consider it as a random variable with respect to the measure $\mu$. For 
$k \in \mathbb{Z}$, the $k^{t h}$ moment of $L$ with respect to the measure $\mu$ is then

$$
M_{k}(X)=\left(L_{*} \mu\right)\left(x^{k}\right)=\int_{X} L^{k}(x) d \mu .
$$

In particular $M_{0}(X)=\mu(X)$. Also the measurable decomposition $X=Z \cup$ $\bigcup_{i} X_{i}$ gives us a formula

$$
M_{k}(X)=\sum_{i} \int_{X_{i}} L^{k}(v) d \mu .
$$

In each identity it is easy to again show that each integral in the summation on the right only depends on the spectrum associated with the identity. Therefore one can find smooth functions $F_{k}$ such that

$$
M_{k}(X)=\sum_{l \in S} F_{k}(l)
$$

where $S$ denotes the spectrum of the given identity. In particular, this formula is the original identity in the case $k=0$. Also the average length of the geodesic associated with an element of $X$, called the average hitting time $A(X)$, is then given by

$$
A(X)=\frac{M_{1}(X)}{\mu(X)}=\frac{M_{1}(X)}{M_{0}(X)} .
$$

\subsection{Moments of Bridgeman-Kahn Identity}

In a recent paper, we consider the moments of the Bridgeman-Kahn identity and show that both the Bridgeman-Kahn and Basmajian identities arise as identities for its moments. The Bridgeman-Kahn identity obviously arises as the identity for the $k=0$ moment. We show that the Basmajian identity appears as the identity for the $k=-1$ moment, giving a link between the two identities. We also derive an integral formula for the moments and an explicit formula for $A(X)$ in the surface case.

Theorem 6.1. (Bridgeman-Tan, [14]) There exists smooth functions $F_{n, k}$ : $\mathbb{R}_{+} \rightarrow \mathbb{R}_{+}$and constants $C_{n}>0$ such that if $X$ is a compact hyperbolic $n$ manifold with totally geodesic boundary $\partial X \neq \emptyset$, then

(1) The moment $M_{k}(X)$ satisfies

$$
M_{k}(X)=\sum_{l \in L_{X}} F_{n, k}(l)
$$

(2) $M_{0}(X)=\operatorname{Vol}\left(T_{1}(X)\right)$ and the identity is the Bridgeman-Kahn identity. 
(3) $M_{-1}(X)=C_{n} \cdot \operatorname{Vol}(\partial X)$ and the identity for $M_{-1}(X)$ is the Basmajian identity.

(4) The average hitting time $A(X)$ satisfies

$$
A(X)=\frac{1}{\operatorname{Vol}\left(T_{1}(X)\right)} \sum_{l \in L_{X}} F_{n, 1}(l)=\sum_{l \in L_{X}} G_{n}(l) .
$$

In the surface case we obtain an explicit formula for the function $G_{2}$ and hence $A(X)$ in terms of polylogarithms. Furthermore, besides compact surfaces obtained as quotients of Fuchsian groups, the identity holds more generally for finite area surfaces with boundary cusps.

Theorem 6.2. (Bridgeman-Tan, [14]) Let $S$ be a finite area hyperbolic surface with non-empty totally geodesic boundary. Then

$$
A(S)=\frac{1}{2 \pi \operatorname{Area}(S)}\left(\sum_{l \in L_{S}} F\left(\operatorname{sech}^{2} \frac{l}{2}\right)+6 \zeta(3) C_{S}\right)
$$

where

$$
\begin{gathered}
F(a)=-12 \zeta(3)-\frac{4 \pi^{2}}{3} \log (1-a)+6 \log ^{2}(1-a) \log (a)-4 \log (1-a) \log ^{2}(a) \\
-8 \log \left(\frac{a^{2}}{1-a}\right) L i_{2}(a)+24 L i_{3}(a)+12 L i_{3}(1-a),
\end{gathered}
$$

for $L i_{k}(x)$ the $k^{\text {th }}$-polylogarithm function, and $\zeta$ the Riemann $\zeta$-function.

\subsection{Moments of Basmajian Identity}

In the recent preprint [50], Vlamis considers the moments for the Basmajian identity.

Theorem 6.3. (Vlamis, [50]) Let $X$ be a compact hyperbolic manifold with totally geodesic boundary and $m_{k}(X)$ be the moments of the boundary hitting function with respect to lebesgue measure on $\partial M$. Then

$$
m_{k}(X)=\sum_{l \in L_{X}} f_{n, k}(l)
$$

where

$$
f_{n, k}(l)=\Omega_{n-2} \int_{0}^{\log \operatorname{coth}(l / 2)}\left(\log \left(\frac{\operatorname{coth} l+\cosh r}{\operatorname{coth} l-\cosh r}\right)\right)^{k} \sinh ^{n-2} r d r
$$

and $\Omega_{n-2}$ is the volume of the unit $(n-2)$-dimensional sphere. 
Vlamis derives an explicit formula in odd-dimensions and further derives a formula for the first moment in the case of a surface $S$.

Theorem 6.4. (Vlamis, [50]) Let $S$ be a compact hyperbolic surface with non-empty totally geodesic boundary. Then

$$
A(S)=\frac{1}{2 \pi \operatorname{Area}(S)} \sum_{l \in L_{S}}\left(L i_{2}\left(-\tanh ^{2} \frac{l}{2}\right)-L i_{2}\left(\tanh ^{2} \frac{l}{2}\right)+\pi^{2} / 4\right) .
$$

\section{The Bowditch proof of the McShane identity and generalizations}

Bowditch gave an algebraic-combinatorial proof of the original McShane identity for the punctured torus in [8], and extending the method, proved variations for punctured torus bundles in $[9$ and representations of the punctured torus group (including the quasi-fuchsian representations) satisfying some conditions he calls the Q-conditions in [10. One advantage of the proof is that it avoids the use of the Birman-Series result on the Hausdorff dimension of the set of points on simple geodesics on a hyperbolic surface. Akiyoshi, Miyachi and Sakuma refined the identity for punctured torus groups in [1] and found variations for quasi-fuchsian punctured surface groups in [2].

Let $T$ be a once punctured hyperbolic torus, and $\pi:=\pi(T)=\langle X, Y\rangle$ the fundamental group of $T$, a free group on two generators, and $\rho: \pi \rightarrow \operatorname{PSL}(2, \mathbb{R})$ the holonomy representation. Define an equivalence relation $\sim$ on $\pi$, by $X \sim Y$ if $X$ is conjugate to $Y$ or $Y^{-1}$. The classes correspond to free homotopy classes of closed curves on $T$, let $\Omega \subset \pi / \sim$ be the set of classes corresponding to essential, simple, non-peripheral simple closed curves on $T$. Classes in $\Omega$ have representatives which form part of a generating pair for $\pi$, we call them primitive classes. For $X \in \Omega, x:=\operatorname{tr} \rho(X)$ is well defined up to sign, and is related to the length $l$ of the unique geodesic representing the class by

$$
\cosh ^{2}(l / 2)=x^{2} / 4 .
$$

McShane's orginal identity for the once-punctured torus then has the form

$$
\sum_{X \in \Omega} h(x)=\sum_{X \in \Omega}\left(1-\sqrt{1-\frac{4}{x^{2}}}\right)=\frac{1}{2}
$$

where we let $x=\operatorname{tr} \rho(X)$, and $h(x)=1-\sqrt{1-\frac{4}{x^{2}}}$. This is the form which was proven by Bowditch, and generalized to type-preserving representations (i.e., $\left.\operatorname{tr} \rho\left(X Y X^{-1} Y^{-1}\right)=-2\right)$ of $\pi$ into $\operatorname{SL}(2, \mathbb{C})$ satisfying the following conditions which he calls the $\mathrm{Q}$ - conditions, we call here the BQ-conditions: 
Definition 7.1. A represention $\rho$ from $\pi$ into $\operatorname{SL}(2, \mathbb{C})$ satisfies the Bowditch Q-conditions (BQ-conditions) if

(1) $\operatorname{tr} \rho(X) \notin[-2,2]$ for all $X \in \Omega$;

(2) $|\operatorname{tr} \rho(X)| \leq 2$ for only finitely many (possibly none) $X \in \Omega$.

The basic idea of the proof was to represent the values taken by elements of $\Omega$ in an infinite trivalent tree. This arises from the fact that $\Omega$ can be identified with $\mathbb{Q} \cup \infty$ by considering the slopes of the curves in $T$, and the action of the mapping class group of $T$ on this set is essentially captured by the Farey tessellation, whose dual is an embedded infinite trivalent tree in $\mathbb{H}$. In this way, $\Omega$ is identified with the set of complementary regions of the dual tree, and the values $\operatorname{tr} \rho(X)$ for $X \in \Omega$ satisfy vertex and edge relations which come from the Fricke trace identities. Using this function and the vertex and edge relations, Bowditch was able to cleverly assign a function on the directed edges of the tree which satisfied some simple conditions and applied a Stoke's theorem type argument to prove the identity.

Subsequently, Tan, Wong and Zhang extended the Bowditch method to prove versions of the identity for general representations of the free group $\pi$ into $\operatorname{PSL}(2, \mathbb{C})$ satisfying the Q-conditions and closed hyperbolic three manifolds obtained from hyperbolic Dehn surgery in [47, 46. Specifically, let $\rho \in \operatorname{Hom}(\pi, \operatorname{SL}(2, \mathbb{C}))$ be a representation satisfying the BQ-conditions and let $\tau=\operatorname{tr} \rho\left(X Y X^{-1} Y^{-1}\right)$. We call $\rho$ a $\tau$-representation. Define the function $\mathfrak{h}:=\mathfrak{h}_{\tau}$ as follows:

For $\tau \in \mathbf{C}$, set $\nu=\cosh ^{-1}(-\tau / 2)$. We define

$$
\mathfrak{h}=\mathfrak{h}_{\tau}: \mathbf{C} \backslash\{ \pm \sqrt{\tau+2}\} \rightarrow \mathbf{C}
$$

by

$$
\begin{aligned}
\mathfrak{h}(x) & =2 \tanh ^{-1}\left(\frac{\sinh \nu}{\cosh \nu+e^{l(x)}}\right) \\
& =\log \frac{e^{\nu}+e^{l(x)}}{e^{-\nu}+e^{l(x)}} \\
& =\log \frac{1+\left(e^{\nu}-1\right) h(x)}{1+\left(e^{-\nu}-1\right) h(x)},
\end{aligned}
$$

where 7.2 , (7.3) and (7.4) are equivalent (see [47] for details). We have:

Theorem 7.2. (Tan-Wong-Zhang, Theorem 2.2, 4 77) Let $\rho: \pi \rightarrow \operatorname{SL}(2, \mathbb{C})$ be a $\tau$-representation (where $\tau \neq 2$ ) satisfying the $B Q$-conditions. Set $\nu=$ $\cosh ^{-1}(-\tau / 2)$. Then

$$
\sum_{X \in \Omega} \mathfrak{h}(x)=\nu \quad \bmod 2 \pi i,
$$

where the sum converges absolutely, and $x:=\operatorname{tr} \rho(X)$. 
When $\rho$ arises as the holonomy of a hyperbolic structure on a one-hole torus with geodesic boundary, or with a cone point, then the above is equivalent to the Mirzakhani [37] and the Tan-Wong-Zhang [45] variations of the McShane identity.

Recently, revisiting the original Bowditch proof, Hu, Tan and Zhang proved new variations of the identity for representations of $\pi$ into $\operatorname{PSL}(2, \mathbb{C})$ satisfying the BQ-conditions [21], we have:

Theorem 7.3. (Hu-Tan-Zhang, [21]) Let $\rho: \pi \rightarrow \operatorname{SL}(2, \mathbb{C})$ be a $\tau$-representation (where $\tau \neq 2$ ) satisfying the BQ-conditions. Let $\mu=\tau+2$. Then

$$
\sum_{X \in \Omega} g(x)=\sum_{X \in \Omega}\left(1-\frac{3 x^{2}-2 \mu}{3\left(x^{2}-\mu\right)} \sqrt{1-\frac{4}{x^{2}}}\right)=\frac{1}{2},
$$

where the sum converges absolutely, and $x:=\operatorname{tr} \rho(X)$.

Note that the type-preserving case occurs when $\tau=-2$ so $\mu=0$ and the above reduces to the original McShane identity in this case.

They also extended this in 22 to identities for orbits of points in $\mathbb{C}^{n}$ under the action of the Coxeter group $G_{n}$ generated by $n$-involutions which preserve the varieties defined by the Hurwitz equation. At the moment, it is not clear what is the underlying geometric interpretation of these identities.

\section{Concluding remarks}

We have shown that the identities obtained by Basmajian, McShane, BridgemanKahn and Luo-Tan are obtained by considering decompositions of certain sets $X$ with finite measure $\mu$ associated to the manifold $M$ obtained by considering some kind of geodesic flow, either from the boundary, or from the interior. Typically, there is a subset of measure zero which is complicated but which does not contribute to the identity, and the subsets of non-zero measure in the decomposition are indexed by some simple geometric objects on the manifold. The measures of each subset typically depends only on the local geometry and data, and not on the global geometry of $M$, which may be easy or fairly complicated to compute. One can apply this general philosophy to try to obtain other interesting identities. There are also many other interesting directions for further research and exploration in this area, we list a number of them below:

(1) Find good applications for the identities, for example use them to say something about the moduli space of hyperbolic surfaces. The McShaneMirzakhani identity was an important ingredient in the work of Mirzakhani in the study of the Weil-Petersson geometry of the moduli space 
of bordered Riemann surfaces. It would be interesting to find similar applications for the Basmajian, Bridgeman and Luo-Tan identities.

(2) Generalize the McShane/Luo-Tan identities to hyperbolic manifolds of higher dimension or to translation surfaces.

(3) The Basmajian and Bridgeman-Kahn identities do not generalize easily to complete finite volume hyperbolic surfaces with cusps (or cone points) as in this case the limit set does not have measure zero. It would be interesting to find other interesting decompositions of horocycles which would generalize these identities, some progress on this has been made recently by Basmajian and Parlier 4 .

(4) It would be interesting to extend the Bowditch method to general surfaces of genus $g$ with $n$ cusps, some progress has been been recently by Labourie and the second author, [25].

(5) Give a geometric interpretation of the variation of the McShane identity (Theorem 7.3 obtained by Hu-Tan-Zhang using the extension of the Bowditch method, and also the identity for the $n$-variables case.

(6) Extend the identities to other interesting representation spaces, see for example the work of Labourie and McShane in 24, and Kim, Kim and Tan in 23.

(7) Extend the moment generating point of view, and derive formulae for the moments of the McShane and Luo-Tan identities.

(8) Obtain identities for general closed hyperbolic manifolds.

(9) Analyze the asymptotics of the functions $f$ and $g$ in the Luo-Tan identity and use it to study the asymptotics of embedded simple surfaces in a hyperbolic surface. 


\section{References}

[1] H. Akiyoshi, H. Miyachi and M. Sakuma, A refinement of McShane's identity for quasifuchsian punctured torus groups, In the Tradition of Ahlfors and Bers, III. The Ahlfors-Bers Colloquium, Oct. 2001, Univ. of Connecticut at Storrs, W. Abikoff, A. Haas (Eds.), Contemporary Mathematics, 355, 21 - 40, 2004.

[2] H. Akiyoshi, H. Miyachi and M. Sakuma, Variations of McShane's identity for punctured surface groups, London Mathematical Society Lecture Notes, Y. Minsky, M. Sakuma 8 C. Series (Eds.), Cambridge University Press, 151-185, 2006.

[3] A. Basmajian, The orthogonal spectrum of a hyperbolic manifold, American Journal of Mathematics, 115 1139-1159, 1993.

[4] A. Basmajian, H. Parlier, private communication.

[5] A. Beardon, The Geometry of Discrete Groups, Springer-Verlag, 1995

[6] J. Birman, C. Series, Geodesics with bounded intersection number on surfaces are sparsely distributed. Topology 24 (1985), 217-225.

[7] F. Bonahon, The geometry of Teichmüller space via geodesic currents, Invent. Math. 92 (1988), 139-162.

[8] B. Bowditch, A proof of McShane's identity via Markov triples, Bull. London Math. Soc., 28 (1996) 73-78.

[9] B. Bowditch, A variation of McShane's identity for once-punctured torus bundles, Topology , 36 (1997) 325-334.

[10] B. Bowditch, Markoff triples and quasi-Fuchsian groups, Proc. London Math. Soc. , 77 (1998) 697-736.

[11] M. Bridgeman, Orthospectra and Dilogarithm Identities on Moduli Space. Geometry and Topology, Volume 15, Number 2, 2011

[12] M. Bridgeman, D. Dumas, Distribution of intersection lengths of a random geodesic with a geodesic lamination. Ergodic Theory and Dynamical Systems, 27(4), 2007

[13] M. Bridgeman, J. Kahn, Hyperbolic volume of n-manifolds with geodesic boundary and orthospectra. Geometric and Functional Analysis, Volume 20(5), 2010

[14] M. Bridgeman, S. P. Tan, Moments of the boundary hitting function for the geodesic flow on a hyperbolic manifold. preprint 2013

[15] D. Calegari, Bridgeman's orthospectrum identity, Topology Proceedings, 38, 173-179, 2011

[16] D. Calegari, Chimneys, leopard spots, and the identities of Basmajian and Bridgeman, Algebraic and Geometric Topology, 10(3), 1857-1863, 2010

[17] J. Dupont, The dilogarithm as a characteristic class for flat bundles. J. Pure Appl. Algebra, 44(1987), no. 1-3, 137-164.

[18] W. Goldman, G. Margulis, Y. Minsky, Complete flat Lorentz 3-manifolds and laminations on hyperbolic surfaces, in preparation 
[19] H. Hu, Identities on hyperbolic surfaces, group actions, the Markoff and Hurwitz equations, $\mathrm{PhD}$ thesis, National University of Singapore, in preparation.

[20] H. Hu and S.P. Tan, Dilogarithm identities for small hyperbolic surfaces, in preparation

[21] H. Hu, S.P. Tan, Y. Zhang, New variations of the McShane identity, in preparation

[22] H. Hu, S.P. Tan, Y. Zhang Coxeter group actions on $\mathbb{C}^{n}$ preserving the Hurwitz equation - dynamics and identities, in preparation

[23] I. Kim, J. Kim, S. P. Tan, McShane's Identity in Rank One Symmetric Spaces eprint arXiv:1203.5901

[24] F. Labourie, G. McShane, Cross ratios and identities for higher TeichmüllerThurston theory, Duke Math. J. 149 (2009), no. 2, 279-345

[25] F. Labourie, S.P. Tan, Extension of Bowditch's proof of McShane's identity to higher genus. in preparation.

[26] D. Lee, M. Sakuma, A variation of McShane's identity for 2-bridge links, to appear, Geometry and Topology.

[27] L. Lewin. Structural Properties of Polylogarithms, Mathematical Surveys and Monographs, AMS, Providence, RI, 1991.

[28] F. Luo, S. Tan, A dilogarithm identity on Moduli spaces of curves, to appear, J. Differential Geom.

[29] F. Luo, S. Tan, A dilogarithm identity on moduli spaces of compact surfaces with boundary and non-orientable surfaces, in preparation

[30] H. Masai, G. McShane, Equidecomposability, volume formulae and orthospectra, preprint 2013

[31] B. Maskit, Kleinian Groups, Graduate Texts in Mathematics, Springer-Verlag, 1987.

[32] G. McShane, A remarkable identity for lengths of curves, Phd. Thesis, Warwick, 1991.

[33] G. McShane, Simple geodesics and a series constant over Teichmuller space, Invent. Math. 132 (1998), no. 3, 607-632.

[34] G. McShane, Weierstrass points and simple geodesics, Bull. London Math. Soc. 36 (2004), no. 2, 181-187.

[35] G. McShane, Simple geodesics on surfaces of genus 2, Annales Academiae Scientiarum Fennicae Mathematica 31 2006, 31-38.

[36] M. Mirzakhani, Simple geodesics and Weil-Petersson volumes of moduli spaces of bordered Riemann surfaces, Phd. Thesis, Harvard, 2005.

[37] M. Mirzakhani, Simple geodesics and Weil-Petersson volumes of moduli spaces of bordered Riemann surfaces, Invent. Math. 167 (2007), no. 1, 179-222.

[38] P. Nicholls. The Ergodic Theory of Discrete Groups, volume 143 of London Mathematical Society Lecture Note Series. Cambridge University Press, Cam- 
bridge, 1989 .

[39] P. Norbury Lengths of geodesics on non-orientable hyperbolic surfaces, Geometriae Dedicata 134 (2008), 153-176.

[40] A. Papadopoulos, G. Théret, Shortening all the simple closed geodesics on surfaces with boundary, Proc. Amer. Math. Soc. 138 (2010), 1775-1784

[41] J. Parkkonen, F. Paulin, Counting common perpendicular arcs in negative curvature. preprint 2013

[42] H. Parlier, Lengths of geodesics on Riemann surfaces with boundary, Ann. Acad. Sci. Fenn. Math. 30 (2005), no. 2, 227-236.

[43] L.J. Rogers. On Function Sum Theorems Connected with the Series $\sum_{1}^{\infty} \frac{x^{n}}{n^{2}}$ Proc. London Math. Soc. 4, 169-189, 1907

[44] D. Sullivan, The density at infinity of a discrete group of hyperbolic motions, Publ. Math. IHES, 50 (1979), pp. 171-202.

[45] S.P. Tan, Y.L. Wong and Y. Zhang, Generalizations of McShane's identity to hyperbolic cone-surfaces, J. Differential Geom. 72 (2006), no. 1, 73-112.

[46] S.P. Tan, Y.L. Wong and Y. Zhang, Necessary and sufficient conditions for McShane's identity and variations, Geom. Dedicata 119 (2006), 119-217.

[47] S.P. Tan, Y.L. Wong and Y. Zhang, Generalized Markoff maps and McShane's identity, Advances in Mathematics, 217 (2008) 761-813.

[48] S.P. Tan, Y.L. Wong and Y. Zhang, McShane's identity for classical Schottky groups, Pacific J. Math. 237 (2008) 183-200.

[49] S.P. Tan, Y.L. Wong and Y. Zhang, End invariants for SL(2, C) characters of the one-holed torus, Amer. J. Math. 130 (2008) 385-412.

[50] N. Vlamis, Moments of a Length Function on the Boundary of a Hyperbolic Manifold. preprint 2013

[51] Y. Zhang, Hyperbolic cone-surfaces, generalized Markoff maps, Schottky groups and McShane's identity, Ph.D. Thesis, National University of Singapore, 2004. 